\title{
Small-scale instabilities of an island wake flow in a rotating shallow-water layer
}

\author{
Samuel Teinturier ${ }^{\mathrm{a}, *}$, Alexandre Stegner ${ }^{\mathrm{a}}$, Henri Didelle $^{\mathrm{b}}$, Samuel Viboud $^{\mathrm{b}}$ \\ a Laboratoire de Météorologie Dynamique, CNRS, ENS, 24 Rue Lhomond, 75005 Paris, France \\ b LEGI/Coriolis, 21 Avenue des Martyrs, 38000 Grenoble, France
}

Unlike the standard two-dimensional Kármán street, the oceanic vortex streets which may occur behind isolated islands are affected by the earth's rotation and the vertical stratification of the thermocline. These effects induce a selective destabilisation of anticyclonic vorticity regions. Several experimental studies were devoted to the inertial instability, which induces transient and three-dimensional perturbations in a rotating fluid layer. However, these previous experiments correspond to a large or finite vertical $h$ to horizontal $L$ aspect ratio $(\alpha=h / L)$ while in an oceanic context this ratio is much smaller than unity ( $\alpha \simeq 0.01$ ). This vertical confinement induces a cutoff vertical scale for unstable perturbations. But, since dissipation preferentially damps smaller scales, the shallowwater aspect ratio $\alpha$ may become so small that no growth will occur. We present here the first experimental investigation of three-dimensional destabilizations of an island wake flow in a shallow-water configuration. These laboratory experiments where performed on the LEGI Coriolis Platform, with small aspect ratio $(\alpha=0.1)$ and large Reynolds numbers $(R e=5000-35,000)$. We have shown that unstable three-dimensional perturbations occur when the island Rossby number $R o=V /\left(\Omega_{0} D\right)$ is large enough $(R o>0.8)$ while the Reynolds number seems to control the duration of this transient instability. Qualitative dye visualisation reveals various types of passive tracer dispersion in the wake. Moreover, according to PIV measurements we have shown that, unlike experiments having large or finite aspect ratio $(\alpha \geq 1)$, the small-scale perturbations do not significantly reduce the local vorticity inside the unstable anticyclone. Hence, the shallow-water configuration

\footnotetext{
* Corresponding author.

E-mail address: samuel.teinturier@polytechnique.edu (S. Teinturier).
} 
( $\alpha \ll 1$ ) seems to reduce the intensity and the impact of threedimensional instabilities in the vortex street. Finally, for high Froude numbers, when the flow becomes supercritical and owing to the generation of large amplitude waves in the wake, the vortex street intensity is strongly reduced.

\section{Introduction}

Island wakes are the location of a strong eddy activity, which have important biological consequences in the formation and transport of organic matter. Strong cyclonic eddies can be recognized in satellite SST image by their cold core of upwelled deep water (Aristegui et al., 1994; Caldeira et al., 2002; Caldeira and Marchesiello, 2002) while anticyclonic vortices or filaments are characterized by warm surface water. Various physical processes such as filamentation, small-scale upwelling or three-dimensional instabilities enhance the horizontal transport and the vertical mixing of nutrientrich deep water when an upper surface current encounter oceanic islands or an archipelago. The role of islands wakes in the biological enrichment of surface waters cannot be neglected (Hasegawa et al., 2004). Motivated by this oceanographic context, we investigated, by means of laboratory experiments, the small-scale and three-dimensional instabilities, which may induce a significant vertical mixing in a rotating shallow-water wake.

It is well known that the rotation alters the stability of incompressible two-dimensional flow with respect to three-dimensional perturbations. The Coriolis force induces a selective destabilisation of anticyclonic vorticity regions. According to linear stability analysis, Johnson (1963) and Yanase et al. (1993) show that three-dimensional unstable modes of parallel shear flow may have stronger growth rates than the standard two-dimensional barotropic modes when the absolute vorticity is negative. This inertial instability also occurs in a geophysical context for rotating, stratified and hydrostatic flows. The linear stability analysis of the primitive hydrostatic equations performed by Emanuel (1979) or by Stevens and Ciesielski (1986) show that inertial modes confined in the anticyclonic vorticity region become unstable when $\Phi_{i}(x, y)=f(f+\zeta)<0$ where $\zeta(x, y)$ is the relative vorticity and $f$ is the Coriolis parameter. Here, again the inertial instability have significantly greater growth rate than the barotropic shear instability. Besides, in the absence of dissipation, the maximum growth rates are reached for infinitesimal vertical wavelength (Stevens and Ciesielski, 1986). Hence, for both the hydrostatic and the non-hydrostatic case, the wavelength selection of the inertial instability is mainly controlled by the dissipative effects (Emanuel, 1979; Yanase et al., 1993; Kloosterziel and Carnevale, 2008).

For circular vortices, the generalized Rayleigh criterion proposed by Kloosterziel and VanHeijst (1991) or Mutabazi et al. (1992) asserts that all anticyclonic vortex columns are unstable to threedimensional perturbations if $\Phi_{R}(r)=\left(f+2 v_{\theta} / r\right)(f+\zeta)<0$ where $v_{\theta}(r)$ is the azimuthal velocity and $\zeta(r)$ the relative vorticity. The WKB methods applied to short vertical waves instability demonstrate that this criterion is indeed a sufficient condition for inviscid three-dimensional instabilities (Sipp and Jacquin, 2000; Billant and Gallaire, 2005). This selective destabilization of intense anticyclones is usually called the centrifugal instability and was also checked by means of laboratory experiments (Kloosterziel and VanHeijst, 1991; Afanasyev and Peltier, 1998) or direct numerical simulations (Bartello et al., 1994; Potylitsin and Peltier, 1999; Kloosterziel et al., 2007). The unstable centrifugal modes, similar to Taylor-Couette rolls, are generally located in an annular region, surrounding the vortex core, where the Rayleigh discriminant $\Phi_{R}$ is negative (Afanasyev and Peltier, 1998; Afanasyev, 2002; Potylitsin and Peltier, 1999; Kloosterziel et al., 2007).

For elliptical vortices (non-axisymmetric structures), a resonant interaction between the inertial waves of the vortex and the underlying strain field could generate three-dimensional perturbations in regions of two-dimensional, elliptical streamlines. The unstable perturbation grows invariably in the vortex core, leading to sinuous undulations of the initial vortex along its vertical axis (Pierrehumbert, 1986; Bayly, 1986). In the presence of rotation, the elliptical instability is weakened for cyclonic vortices and enhanced for strong anticyclones. According to the stability analysis of Cambon et al. (1994), Le Dizès (2000) and Le Bars et al. (2007) anticyclonic elliptical vortex will be the most unstable to 
Table 1

Summary of characteristic length, velocity and Rd scales, and Ro and Bu parameters, for various in situ wakes

\begin{tabular}{llllllll}
\hline References & Island & $U\left(\mathrm{~ms}^{-1}\right)$ & $L(\mathrm{~km})$ & $h(\mathrm{~m})$ & $R d(\mathrm{~km})$ & $R o(U / L f)$ & $B u\left(2 R_{d} / L\right)^{2}$ \\
\hline Heywood et al. (1996) & Aldabra & 0.8 & $15-30$ & 150 & 20 & $1-3$ & $2-7$ \\
Hasegawa et al. (2004) & Aoga-Shima & 1.5 & 3 & 100 & - & 5 & - \\
Coutis and Middleton (1999) & Cato & 0.7 & 20 & 200 & 20 & 0.6 & 4 \\
Caldeira et al. (2005) & Catalina & 0.5 & $1-10$ & 100 & - & $0.5-5$ & - \\
Chavanne (2007), Xie et al. (2001) & Hawaii & 0.2 & $1-150$ & - & - & $0.02-2$ & - \\
\hline
\end{tabular}

core-centred perturbations when $\zeta=-2 f$. Both numerical simulations (Potylitsin and Peltier, 1999) and laboratory experiments (Afanasyev, 2002; Stegner et al., 2005; Le Bars et al., 2007) demonstrate that these core-centred modes could dominate the standard centrifugal modes when the ellipticity is strong enough. For this inertial-elliptical instability, the wavelength selection is strongly controlled by the Rossby number.

A general inviscid criterion which takes into account the inertial, the centrifugal and the elliptical instabilities was introduced by Leblanc and Cambon (1997). According to the short-wave stability theory, a certain class of three-dimensional pressureless modes will become unstable if, in a cartesian coordinate frame, $\Phi_{L C}=-(1 / 2) \underline{S}: \underline{S}+(1 / 4) W_{t} . W_{t}<0$ somewhere in the stationary flow domain. We respectively introduce here the rate-of-strain tensor $\underline{S}$, or in other words the symmetric part of the velocity gradient, and the tilting vorticity $W_{t}=\zeta+4 \Omega_{0}$ (Cambon et al., 1994). The relation between the standard stability criterions, mentioned above, and the sufficient condition $\Phi_{L C}<0$ was extensively discussed for simple plane flows subjected to the Coriolis force in Leblanc and Cambon (1997).

For an island wake in deep water, when an upper surface current encounter an isolated island or an archipelago, the boundary stress associated with the nearshore or the lateral side of the island induce a strong boundary shear flow. In this deep water case the influence of bottom drag becomes negligible, as discussed by Tomczak (1988). Just behind the island, the detachment of the lateral boundary layer first leads to a free shear layers. In a second stage, these parallel shear layers could roll up alternatively on both side of the island into elliptical structures. Then, these transient elliptical structures evolve quickly to axisymmetric vortices and generate a von Kármán vortex street in the lee of the island. Hence, at each stage, specific three-dimensional instabilities (inertial, elliptical or centrifugal) could induce a significant vertical mixing in anticyclonic vorticity regions. However, such type of instabilities will occurs only if the relative vorticity satisfy $\varepsilon=\zeta / f<-1$ somewhere. In situ measurements of the local vorticity in the ocean is a hard and expensive task, which requires intensive ADCP surveys (Hasegawa et al., 2004), such measurements are therefore very seldom. Hence, to estimate the order of magnitude of $\varepsilon$ we generally use the global Rossby number $R o=U / L f$ where $U$ is the characteristic current velocity and $L$ is the typical radius of the island. When this Rossby number get close to unity we could expect that locally the magnitude of the vorticity exceed the Coriolis parameter $|\varepsilon|>1$. According to the data collected in Table 1, this could be the case for various volcanic islands or archipelago such as Aldabra in the Mozambique channel (Heywood et al., 1996), Cato reef along the Australian coast (Coutis and Middleton, 1999), Agoa-Shima south to the Japanese coast (Hasegawa et al., 2004), the Hawaii islands (Xie et al., 2001; Chavanne, 2007), Santa Catalina island (Caldeira et al., 2005), or the Gran Canaria archipelago (Aristegui et al., 1994).

An important parameter for deep island wake is the vertical aspect ratio $\alpha=h / L$ where $h$ is the upper surface current thickness (i.e. the thermocline depth) and $L$ the typical horizontal scale of the island or the eddies. Note that $h \simeq 100-200 \mathrm{~m}$ is generally much smaller than the total water depth $H \simeq 1-2 \mathrm{~km}$ for isolated volcanic islands. According to Table 1 , this aspect ratio parameter is very small $\alpha \simeq 0.01$ in the ocean and we could expect a hydrostatic wake flow. The impact of this vertical confinement $(\alpha \ll 1)$ on three-dimensional small-scale instabilities is poorly known and was never investigated experimentally.

An other parameter which governs the island wake is the Burger number $B u=\left(R_{d} / L\right)^{2}$, this latter is given by the relative island size. Small (large) island in comparison with the local deformation radius $R_{d}$ leads to large (small) Burger number. This parameter also controls the barotropic/baroclinic nature of the wake flow, or, in other words, the repartition between the potential and the kinetic energy of the 
mean flow. For large Burger numbers $\left(L \ll R_{d}\right)$ the total energy is mainly kinetic, while for small values of the Burger number $\left(L \gg R_{d}\right)$ the energy is mainly potential. Besides, for large-scale wakes $(B u \ll 1)$ when the isopycnal deviation become finite, the vortex street could strongly differ from the classical Kármán wake. For some extreme cases in the frontal regime $(B u \leq 0.1, R o \simeq 0.1)$ coherent cyclones do not emerge at all and only an anticyclonic vortex street appears in the lee of the island (Perret et al., 2006b, a). However, to focus this study on small-scale three-dimensional instability we consider only finite Rossby numbers and large enough Burger numbers $(B u \geq 1)$ corresponding to the oceanic cases given Table 1.

A recent study (Dong et al., 2007) investigated numerically the dynamics of hydrostatic threedimensional deep island wakes. The Regional Oceanic Model System (ROMS) was used with a high horizontal $(\delta x=250 \mathrm{~m})$ and vertical $(\delta z=25 \mathrm{~m})$ resolution. Such a resolution was needed to capture the small-scale instabilities which do not appears in previous numerical studies of oceanic wakes. For large enough Reynolds number, when $R o=0.1-0.5, \alpha \simeq 0.01$ and $B u \simeq 1$ the magnitude of the local vorticity exceed unity and small-scale three-dimensional unstable perturbations growth in anticyclonic vorticity regions. The horizontal scale of the perturbation decreases when the Reynolds number or the spatial resolution increases as for the case of inertial or centrifugal instabilities.

In order to study the various three-dimensional instabilities (inertial, elliptical and centrifugal) which destabilize anticyclonic vorticity regions in the wake of an isolated island we performed several laboratory experiments. Unlike numerical simulations solving the hydrostatic primitive equations, non-hydrostatic flow may occurs in the laboratory. To satisfy the shallow-water constraint (small aspect ratio $\alpha \ll 1)$ and to reach a finite Rossby number $R o \simeq 1$ and a large Reynolds number $(R e \geq$ 4000) we performed these experiments on the LEGI-Coriolis platform in Grenoble.

The paper is organized as follows: the experimental setup and measurements techniques are described Section 2; the various dispersion regimes of passive dye tracers are presented in Section 3; quantitative PIV measurements are analysed in Section 4; while discussion and summary are given in Section 5.

\section{Experimental setup and physical parameters}

\subsection{Experimental setup}

In order to study small-scale instabilities induced by the rotation in a shallow-water vortex street, we performed several experiments on the 13-m-diameter rotating platform at the LEGI-Coriolis in Grenoble. The turntable had an anti-clockwise rotation (as the planetary rotation), the angular velocity $\Omega_{0}$ could reach a value up to $2 \mathrm{rpm}$ and the corresponding Coriolis parameter $f=2 \Omega_{0}$ was varied between $f=0.04 \mathrm{rad} \mathrm{s}^{-1}$ and $f=0.08 \mathrm{rad} \mathrm{s}^{-1}$. To reproduce the dynamic of a surface current interacting with an isolated and steep island (Fig. 1 (a)), we used a two-layer stratification and we towed in the upper layer a cylinder of diameter $D=50 \mathrm{~cm}$ and heigth $h_{c}=4 \mathrm{~cm}$ (Fig. 1(b)). We assume that the motion of the cylinder transfer momentum mainly in the upper layer and that the dynamic is governed by the first baroclinic mode. This will be generally the case for a deep lower layer, namely when $h \ll H$. In the experiments we first fill the tank with a deep $H=55 \mathrm{~cm}$ and salty layer $\rho_{2} \simeq 1005 \mathrm{~kg} / \mathrm{l}$. Due to the slow Ekman recirculation, it took 1 day for this thick layer to reach a solid-body rotation. Then, we slowly inject (during few hours) a thin surface layer $h=5 \mathrm{~cm}$ of fresh water $\rho_{1} \simeq 999 \mathrm{~kg} / \mathrm{l}$. In this case the two-layer aspect ratio $\delta=h / H \simeq 0.1$ is small enough and the deep lower layer acts as a neutral layer with a small Ekman number $E k=v / f H^{2} \simeq 10^{-4}$ corresponding to a weak Ekman pumping.

For each experiments, the cylinder was towed at a constant velocity $V$ for values varying from $0.8 \mathrm{~cm} \mathrm{~s}^{-1}$ to $6 \mathrm{~cm} \mathrm{~s}^{-1}$. We introduced a global Rossby $(R o)$ and a Reynolds $(R e)$ numbers for this experimental setup:

$$
R o=\frac{V}{\Omega_{0} D}, \quad R e=\frac{V D}{v}
$$

where $v$ is the kinematic viscosity of water. By changing either the drifting speed $V$ or the angular velocity $\Omega_{0}$ we easily reached finite Rossby numbers $R o=0.4-4$ and large Reynolds numbers $R e=$ 4000-30, 000 . 
(a)

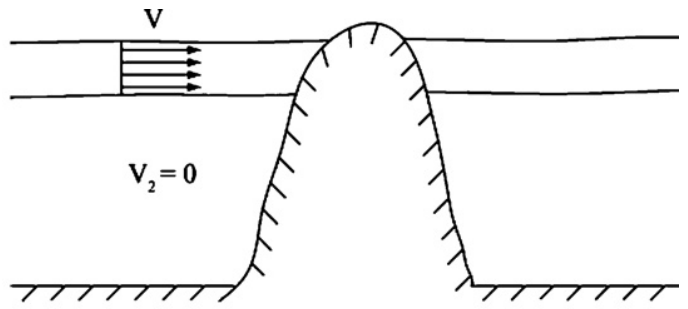

(b)

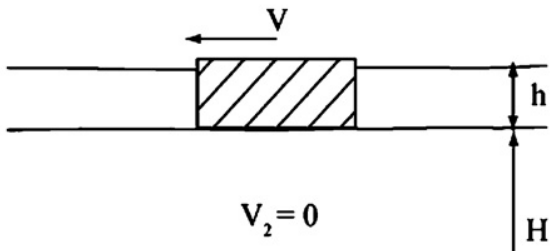

Fig. 1. Oceanic island wake and the laboratory model: (a) oceanic surface current interacting with an isolated island; (b) steady drifting cylinder in the upper layer.

We introduced the aspect ratio parameter:

$$
\alpha=\frac{h}{D}=0.1
$$

which compare the vertical to the horizontal scales in the experiments. Unlike previous experiments on rotating Kármán streets (Boyer and Kmetz, 1983; Tarbouriech and Renouard, 1996; Stegner et al., 2005) where the aspect ratio is large $\alpha \gg 1$ or finite $\alpha \simeq 1$, we investigate in the present study an island wake in a shallow rotating layer where $\alpha \ll 1$. The validity of the rotating shallow-water model for laboratory experiments was discussed in Stegner (2007). If the dimensionless parameters satisfies $R o \alpha^{2} \ll 1$ the slow vortical motion in the thin upper layer is expected to satisfy the shallow-water approximations. Hence, even for finite Rossby number the small aspect ratio $(\alpha \simeq 0.1)$ guarantee the hydrostatic balance of the large-scale flow. However, for small-scale unstable perturbations, the vertical to horizontal ratio could reach unity $(\alpha \simeq 1)$ and non-hydrostatic dynamics could be locally encounter.

\subsection{Two-layer stratification}

Due to the large-scale of the experiment, high Reynolds numbers were reached and therefore the dissipation was weak. In order to avoid residual motion it was then needed to wait at least one or $2 \mathrm{~h}$ between consecutive experiments. Hence, it takes several days to achieve a set of experiments at a given rotation rate $\Omega_{0}$. During that time, both the air friction on the free surface and the molecular diffusion of salt tend to smooth the initial two-layer stratification. We used a conductivity and temperature profiler (125MicroScale model, from PME $^{1}$ ) to follow the evolution of the density stratification in the tank. According to Fig. 2, the upper surface layer becomes linearly stratified within 3 days. To test the impact of this change in stratification on the wake flow, we performed some experiments with the same forcing conditions $\left(V\right.$ and $\Omega_{0}$ ) but different stratifications. We did not observe significant changes in the dynamics or the small-scale instability of the wake flow due to the evolution of the two-layer stratification. Hence, we assume that the we transfer momentum and kinetic energy mainly in the upper layer and that the dynamics is governed by the first baroclinic mode corresponding to an

1 http://www.pme.com. 


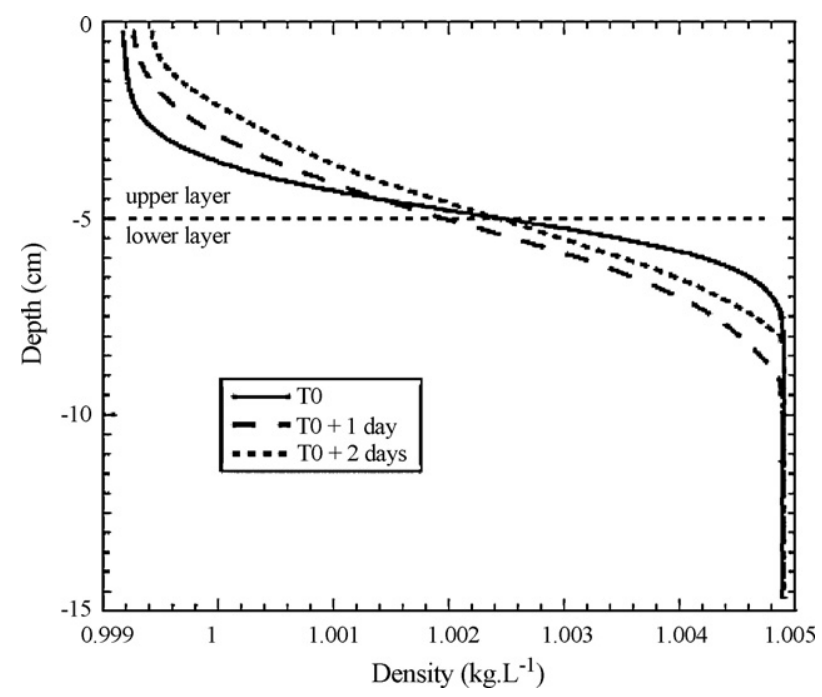

Fig. 2. Evolution of the density profile over 3 days for the first $15 \mathrm{~cm}$ of water.

intense velocity in the thin layer while the deep lower layer remain almost at rest. For a perfect twolayer stratification the deformation radius $R_{d}^{*}$ associated to the first baroclinic component of motion is given by $R_{d}^{*}=\sqrt{g^{*}[H h /(H+h)]} / 2 \Omega_{0}$. In our case, when $h$ is small in comparison to $H$, the baroclinic deformation radius can be approximated by $R_{d}^{*} \simeq \sqrt{g^{*} h} / 2 \Omega_{0}$ where $g^{*}=g\left(\rho_{2}-\rho_{1}\right) / \rho$ is the reduced gravity. If we consider a continuous stratification: linear in the upper layer and constant in the deep lower layer, the deformation radius associated to the first baroclinic mode will remain close to $R_{d}^{*}$. The typical values $R_{d}^{*}=65-125 \mathrm{~cm}$ are slightly larger than the cylinder diameter $D=50 \mathrm{~cm}$ and lead to a large Burger number:

$$
B u=\left(\frac{2 R_{d}^{*}}{D}\right)^{2} .
$$

In the present experiments $B u=6-25$ and therefore, the displacement of the isopycnal interface between the thin surface layer and the deep layer is expected to be small or moderate. However, this interface support internal gravity waves with a maximum phase speed $c=\sqrt{g^{*} h}$. For almost all the experiments, the typical phase speed velocity was about $c=6-7 \mathrm{~cm} \mathrm{~s}^{-1}$ and generally larger than the cylinder velocity $V$. Hence, the Froude number $F_{d}=V / c$ was below unity and the wake flow is expected to be subcritical. However, for very few cases supercritical wakes $F_{d}>1$ were investigated.

We can also define a local Brunt-Vaisala frequency $N^{2}=-\left(g / \rho_{0}\right)(\partial \rho / \partial z)$ with the maximum density gradient 2 . The typical value of $N$ was between 1 and $1.5 \mathrm{~s}^{-1}\left(0.16\right.$ and $\left.0.24 \mathrm{rad} \mathrm{s}^{-1}\right)$ approximately four times $f$. With structures which have a vorticity field $\zeta=\operatorname{rot} v$, we can write a dynamical Froude number comparing $\zeta$ and $N: F r=\zeta / N$.

\subsection{Qualitative dye and quantitative PIV measurements}

Qualitative visualizations of the wake behind the cylindrical island were done using passive dye tracers. Two separated injection holes were made on both side of the cylinder $2 \mathrm{~cm}$ bellow the free surface in order to release continuously the dye in the lateral boundary layers (Fig. 3). The dye density was carefully adjusted according to the upper layer stratification to avoid vertical mixing at the injection. Besides, we carefully adjust the injection rate to the drifting velocity of the cylinder in order to reduce the jet instability, which could occur at the exit of the injection hole. Different colours were 


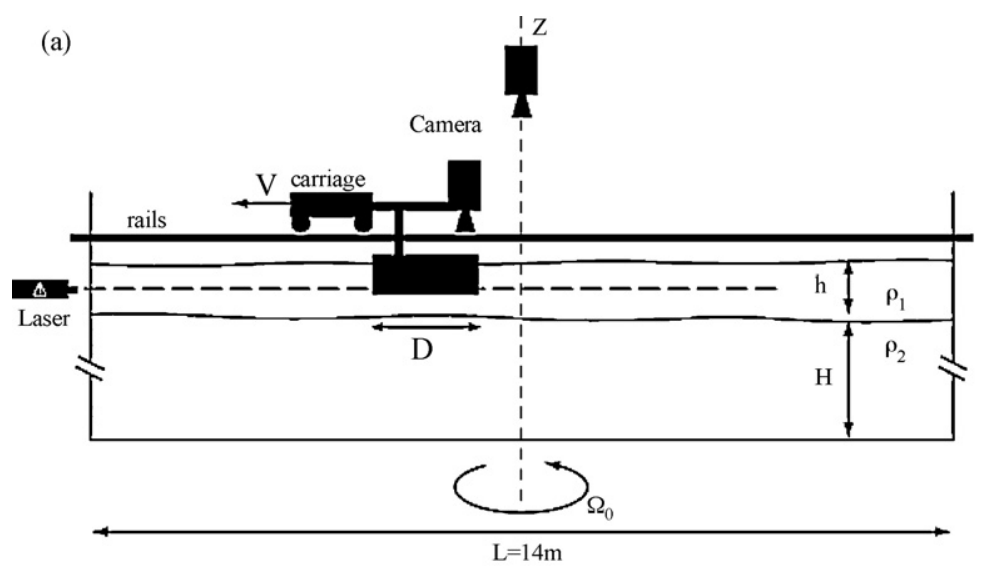

(b)

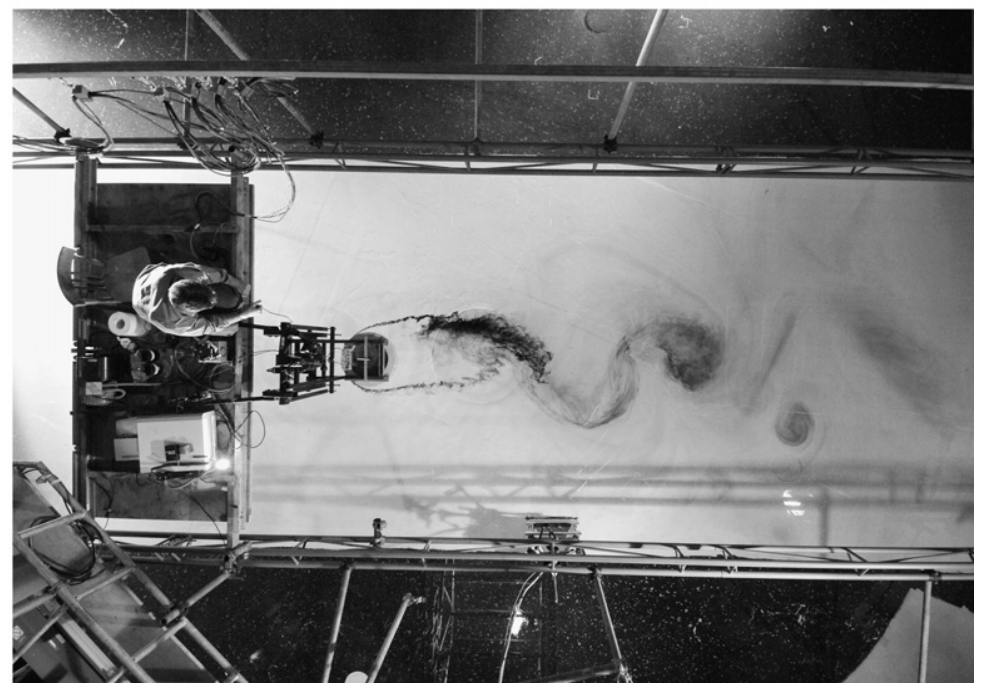

Fig. 3. Side view (a) of the experimental setup and top view, (b) visualization of an experiment.

used on each side of the cylinder: black (red) in the anticyclonic (cyclonic) boundary layer. In a rotating layer, the dye visualization is very sensitive to three-dimensional perturbations, which break the bi-dimensionality of the flow and allow detecting with high sensitivity three-dimensional small-scale instabilities in the cylinder wake. To record the stable or unstable dynamic within the wake we used a DV $640 \times 480$ colour video camera and a $2048 \times 1536$ colour digital camera. Blench was added in the upper layer to dissolve the remaining dye between two consecutive experiments without changing the water.

Standard particle image velocimetry (PIV) was used to measure the horizontal velocity field. Small buoyant particles (200-400 $\mu$ m diameter) were introduced in the upper layer and lightened with 488$\mathrm{nm}$ Argon laser sheet focused $2 \mathrm{~cm}$ below the free surface. In order to avoid sedimentation the particle density was carefully matched with the upper layer density. The particles' motion was recorded by a $1024 \times 1024$ pixels charge-coupled device (CCD) camera. For some experiment we add a second $720 \times 576$ pixels analog video camera on the carriage supporting the cylinder. This second camera allows recording the particles' motion within the boundary layer and just behind the cylinder. The particles' velocity was analyzed using LAVISION PIV software with successive overlap cross-correlation 
boxes yielding a final $70 \times 70$ horizontal velocity field. The velocity grid resolution (distance between two vectors) is about $\delta x=2.4 \mathrm{~cm}$. Vorticity fields were derived from 10 to 25 averaged velocity fields. The averaging period corresponds roughly to $25-50 \mathrm{~s}$, in other words about $1 / 4$ of the turntable rotation period (160s).

\section{Results}

\subsection{Evolution of passive dye tracers}

We first performed several experiments to investigate qualitatively the parameter space $(R o, R e)$ and detect the regions where three-dimensional and small-scale instabilities occur. The constant release of passive dye tracers in the cylinder boundary layer was used to observe the nature of the flow field at a large and a small-scale. Moreover, as far as oceanic island is concerned, the evolution of the dye intensity indicates how a passive tracer (released along the coast) such as a pollutant or biological species could be mixed and dispersed in the island wake. The dispersion or concentration of the passive dye tracers in the wake could be classified in three types: the core vortex concentration (CVC), the core anticyclonic mixing (CAM) and the anticyclonic stretching (AS).

The core vortex concentration corresponds to the evolution of passive tracers in classical twodimensional Kármán street. A typical example is shown in Fig. 4. The cylinder was towed from the right to the left at a constant speed of $V=0.8 \mathrm{~cm} \mathrm{~s}^{-1}$ and due to the anti-clockwise rotation of the platform, the anticyclonic boundary layer and vortices (black dye) are above the cyclonic ones (red dye). In this case $(R o=0.4, R e=4000, B u=6.5)$ the dye tracers remain concentrated in the core of vortices of both sign. The two-dimensional dye patterns are similar in cyclonic and anticyclonic vortices. Due to the rotation and the vertical confinement in the thin upper layer, the wake flow seems to be mainly two-dimensional even at large Reynolds number. Horizontal stretching and small-scale filamentation are observed but there is no visible evidence of three-dimensional motions. The size of the main dye patterns, circular patches centred in the core of vortices is identical to the size of the cylindrical island. The distance $L_{p}$ between two dye patches of same colour, in other words same sign vortices, corresponds roughly to five cylinder diameter. This is in agreement with previous studies (Boyer and Kmetz, 1983; Stegner et al., 2005) indicating that for Kármán vortex street, the eddy shedding at a time interval $\mathrm{T}$ is not affected significantly by the rotation. The Strouhal number remain close to the two-dimensional non-rotating and large Reynolds limit $S t=D /(T U)=D / L_{p} \simeq 0.2-0.25$ (Wen and Lin, 2001).

If we increase the drifting speed and therefore the global Rossby number Ro, three-dimensional perturbations appears in black dye patterns. These small-scale perturbations, in comparison with the vortex size, growth in the core of anticyclonic vorticity regions and we therefore use the term core anticyclonic mixing. A typical example of CAM is shown in Fig. 5 corresponding to a finite Rossby number $R o=1$ with $R e=10,000$ and $B u=6.5$. Hence, as far as the mixing of passive tracers is concerned, a significant cyclone-anticyclone asymmetry appears in the island wake for finite Rossby numbers. Similar dye visualizations of anticyclonic destabilisation and mixing in a rotating Kármán street were made by Boyer and Kmetz (1983) and Stegner et al. (2005). However, in the present case, the aspect ratio parameter is small ( $\alpha \simeq 0.1$ ) and due to the vertical confinement of the thin upper layer we cannot detect any vortex-core undulations along the vertical, as observed by Afanasyev (2002), Stegner et al. (2005) and Le Bars et al. (2007) for rotating experiments with large or finite aspect ratio ( $\alpha \geq 1)$. After few rotation periods, the three-dimensional perturbations slowly disappeared while a circular and diffuse patch of black dye tracer remain visible. This stage corresponds to a relaminarization of the flow. Hence, for a CAM event the distribution of the passive tracer will remain almost circular inside the anticyclonic vortices even if transient three-dimensional mixing occurs in the early stage of shedding.

For larger Reynolds number, small-scale turbulent motions occur in the lateral boundary layers and the near wake just behind the cylinder. Then, further in the wake, a strong cyclone-anticyclone asymmetry of the dye pattern occurs. A typical example of anticyclonic stretching of passive tracers is shown in Fig. 6 corresponding to $R o=2, R e=20,000, B u=7.5$. In this case, the black dye tracer initially released in the anticyclonic boundary layer is strongly stretched and dispersed in the wake. 
(a)

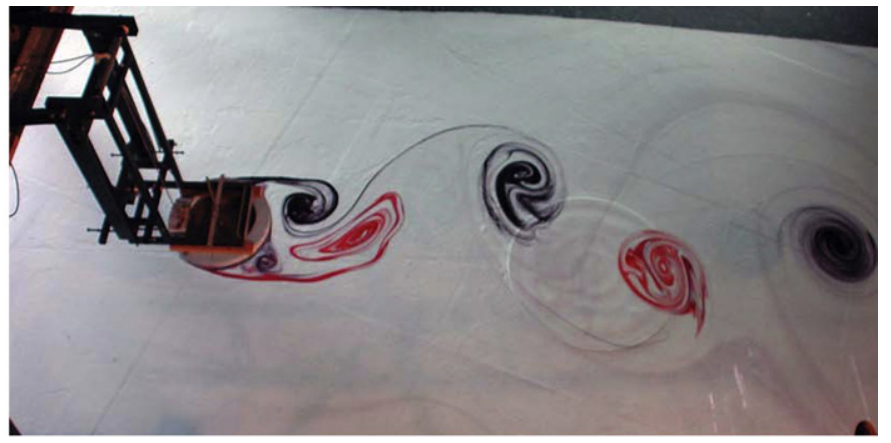

(b)

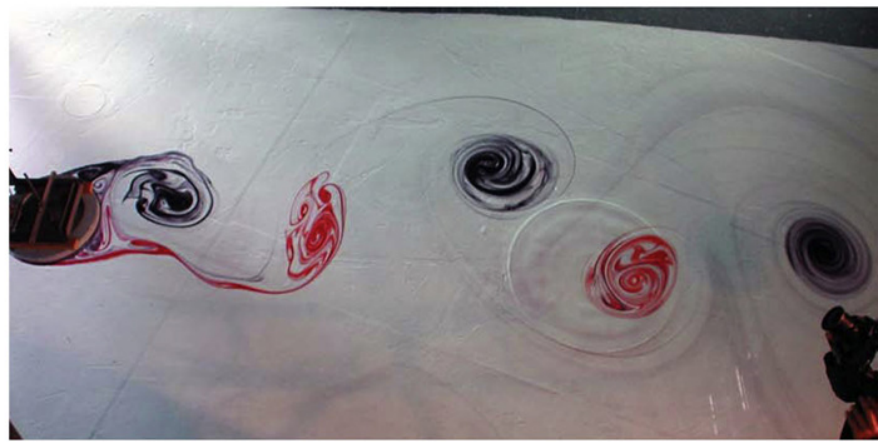

(c)

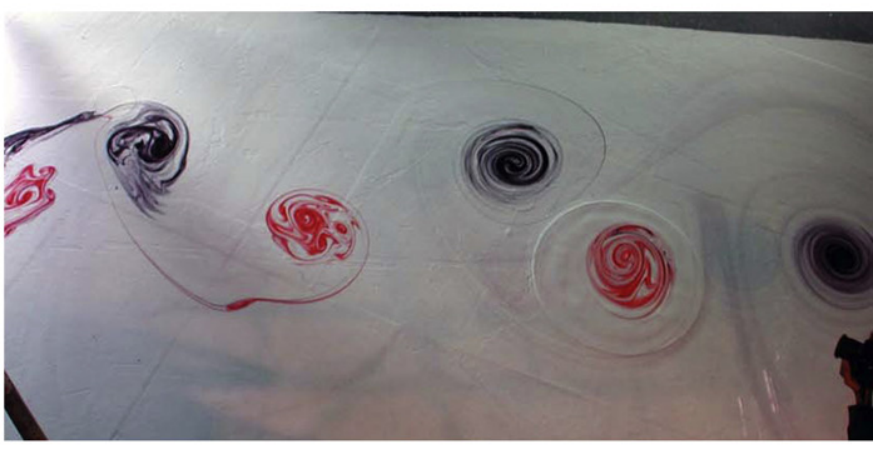

(d)

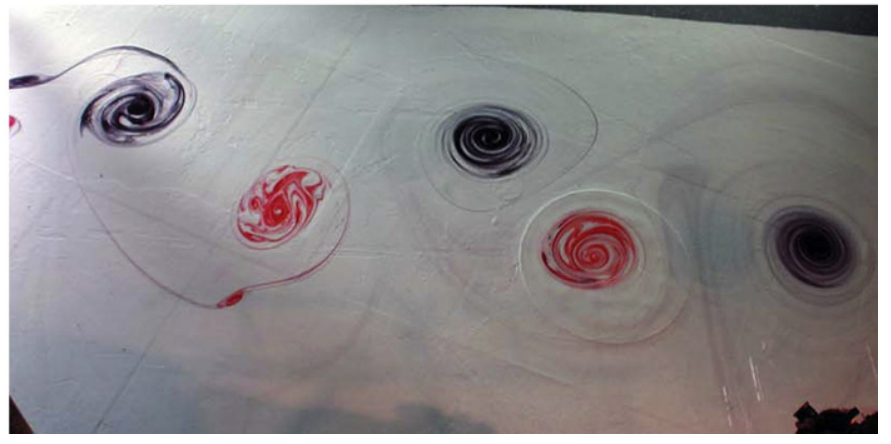

Fig. 4. Top-view vizualisation of black (anticyclonic) and red (cyclonic) dyes released in the boundary layer of the cylinder, for $R o \simeq 0.4, R e \simeq 4000$, and $B u \simeq 6.5$, at (a) $t=0 \mathrm{~s}$, (b) $t \simeq 120 \mathrm{~s}$, (c) $t \simeq 240 \mathrm{~s}$, and (d) $t \simeq 360 \mathrm{~s}$. 


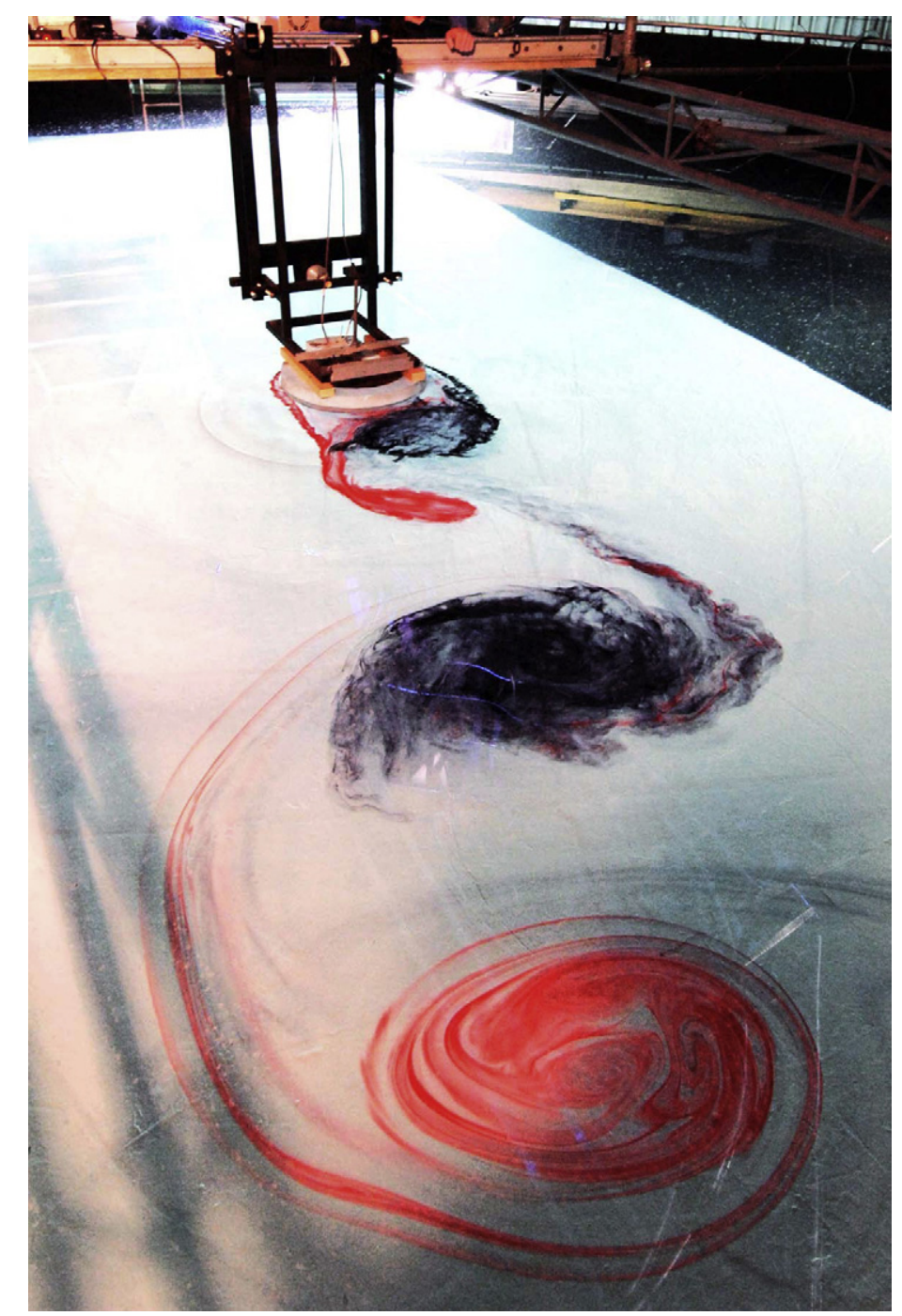

Fig. 5. A front view of a dye experiment for $R o \simeq 1, R e \simeq 10,000$, and $B u \simeq 6.5$.

While, on the other hand, most of the red dye, initially released in the cyclonic boundary layer, remain concentrated in the core of cyclonic vortex. The turbulent boundary layer, which is visible for both tracers, does not seem to affect significantly the final distribution of the red (cyclonic) dye in the island wake.

We plot in Fig. 7 the range of parameter space where the three scenario of passive tracer distribution (CVC, CAM or AS) occurs. Mixing and dispersion in the island wake (confined in an upper shallow-water layer) appears to be controlled by both the Rossby and the Reynolds number. According to previous theoretical (Yanase et al., 1993) and numerical (Kloosterziel et al., 2007; Dong et al., 2007) studies, unstable three-dimensional perturbations should mix the flow when locally the absolute vorticity $\zeta+f$ becomes negative. This should occur for finite Rossby numbers, if the Reynolds number is not too small. However, according to the qualitative nature of the dye visualisations, we cannot define a precise threshold in the Rossby number. Nevertheless, the asymmetry can be easily detected for Ro $\geq 0.7-1$ 
(a)

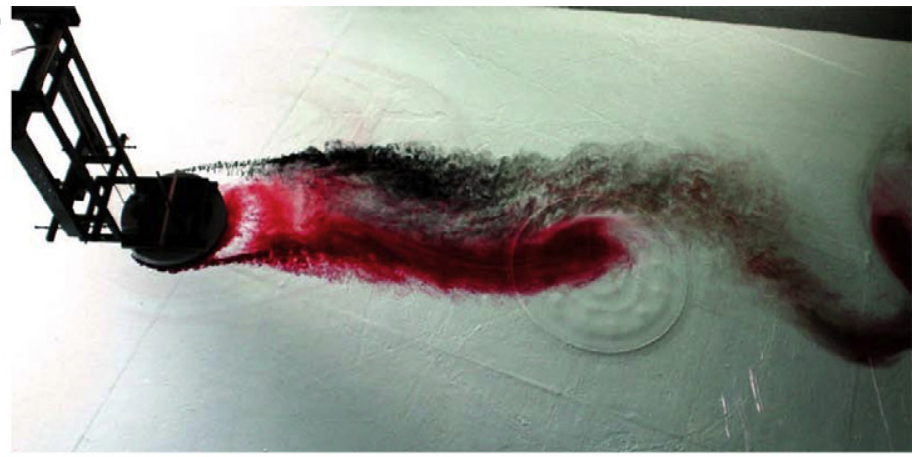

(b)

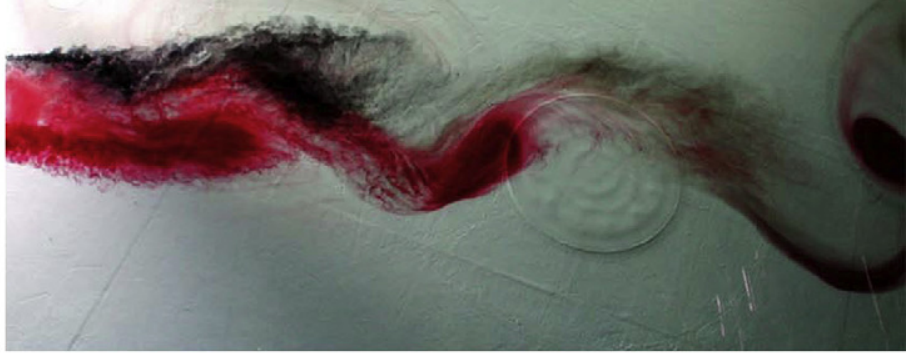

(c)

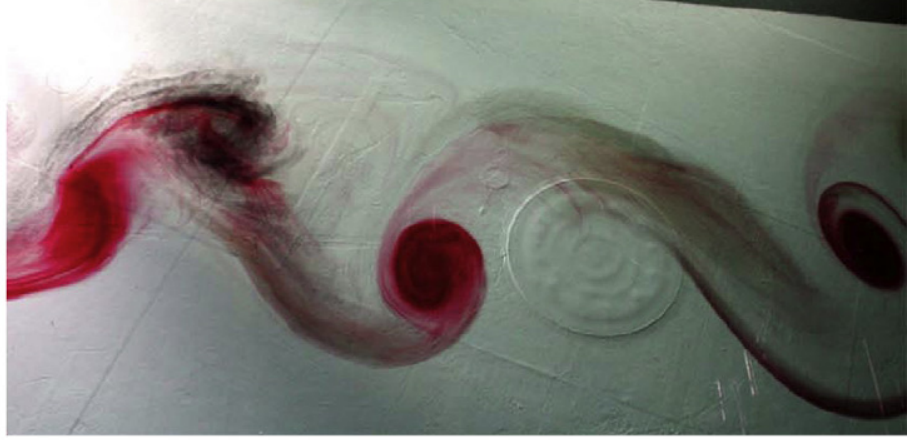

(d)

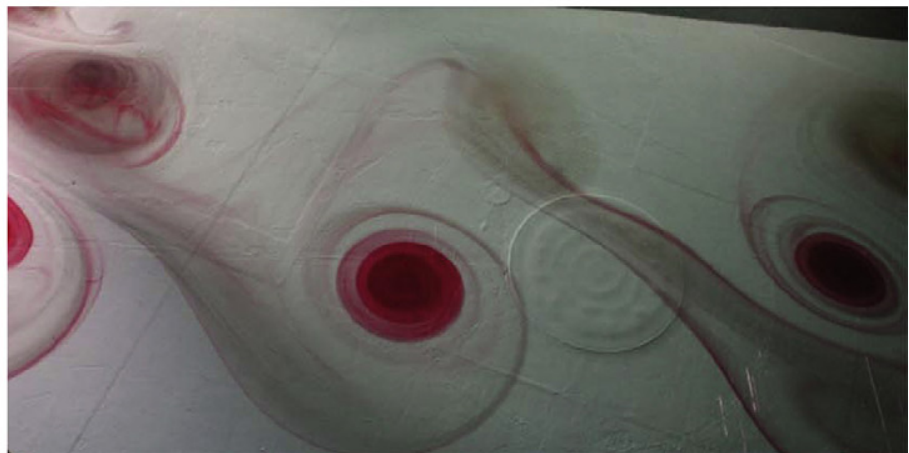

Fig. 6. Top-view of dye experiment, for $R o \simeq 2, R e \simeq 20,000$, and $B u \simeq 7.5$, at (a) $t=0 \mathrm{~s}$, (b) $t \simeq 30 \mathrm{~s}$, (c) $t \simeq 60 \mathrm{~s}$, and (d) $t \simeq 300 \mathrm{~s}$. 


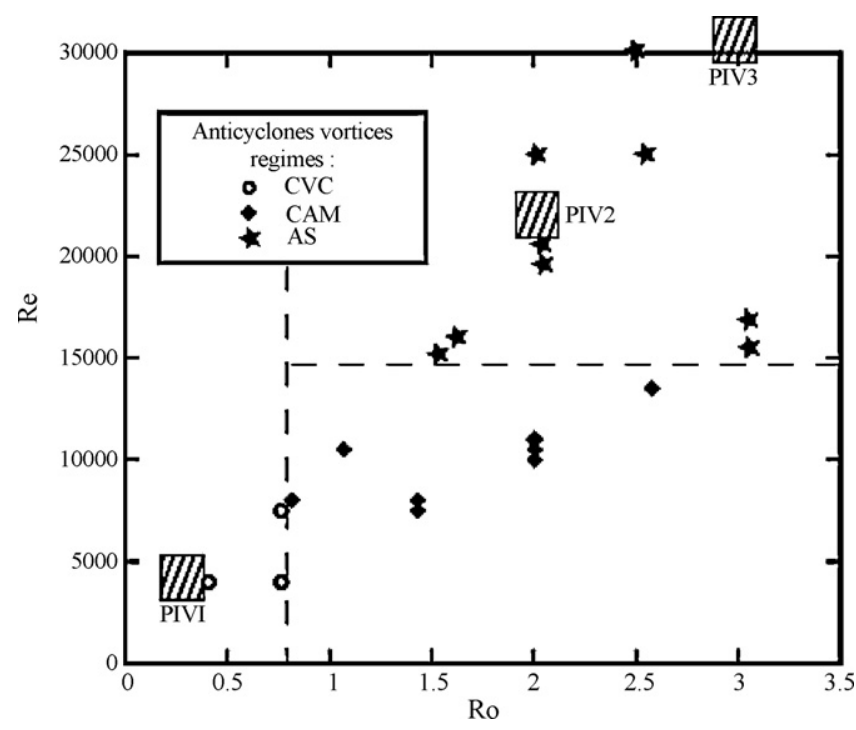

Fig. 7. Parameter space diagram $(R o, R e)$ of the various dye patterns CVC, CAM or AS observed in the rotating shallow-water wake. The aspect ratio parameter $\alpha$ is fixed for all experiments $(\alpha=0.1)$, the Burger number $B u$ is $B u=6$ or $B u=20$, depending on the turntable angular velocity. Three regions are qualitatively delimited with dashed line. The three large dashed square indicates the parameters of the PIV measurements analysed in Section 3.2.

and we draw in Fig. 7 a dashed line as an approximate boundary at $R o \simeq 0.8$ between CVC and CAM or AS patterns. The Reynolds number mainly controls the separation between the core anticyclonic mixing and the strong anticyclonic stretching. Here again, we draw an approximate boundary in dashed line for $R e \simeq 15,000$. This large Reynolds value depends probably on the strong vertical confinement $\alpha=0.1$. Indeed, according to Fig. 12 in Stegner et al. (2005), typical AS patterns occurs for much smaller Reynolds number $(R e \simeq 150)$ when the aspect ratio parameter is finite $\alpha=1$.

For standard two-dimensional Kármán streets, the region of high concentration of dye indicate the vortex centre (i.e. vorticity extrema) in the wake. In the present study, when three-dimensional perturbations strongly mix and diffuse passive tracers the relation between the dye pattern and an active tracer such as vorticity is not obvious. Hence, as far as the dynamical evolution of the wake is concerned, passive tracer observations should be taken with care. Therefore, in order to complement these qualitative dye visualizations PIV measurements are needed to quantify accurately the size and the intensity of the eddies in the lee of the cylindrical island.

\subsection{Particle image velocimetry measurements}

Few experiments using standard particle image velocimetry were done to measure the horizontal velocity field in the thin upper layer. We focused our efforts to accurately quantify the vorticity field. Unfortunately, due to experimental limitations and unexpected particles sedimentation, we were able to measure the vorticity and follow the dynamics only in very few experiments. Four cases are presented bellow: a standard Kármán wake at small Rossby number (PIV1), a finite Rossby number wake (PIV2), a specific case of supercritical wake corresponding to a wave-vortex wake (PIV3) and a zoomed visualization of the anticyclonic detached boundary layer at finite Rossby number (DBL).

\subsubsection{Small Rossby number wake (PIV1)}

In a first step, we measured the vorticity field for a small Rossby number regime $(R o=0.4, R e=$ $4000, B u=6.5$ ) corresponding to a standard two-dimensional Kármán street and a CVC dye pattern (Fig. 4) described in the previous section. The dynamical evolution of the vorticity field in the near wake is shown in Fig. 8. In this top view measurement, the cylinder moves from top to bottom. The 

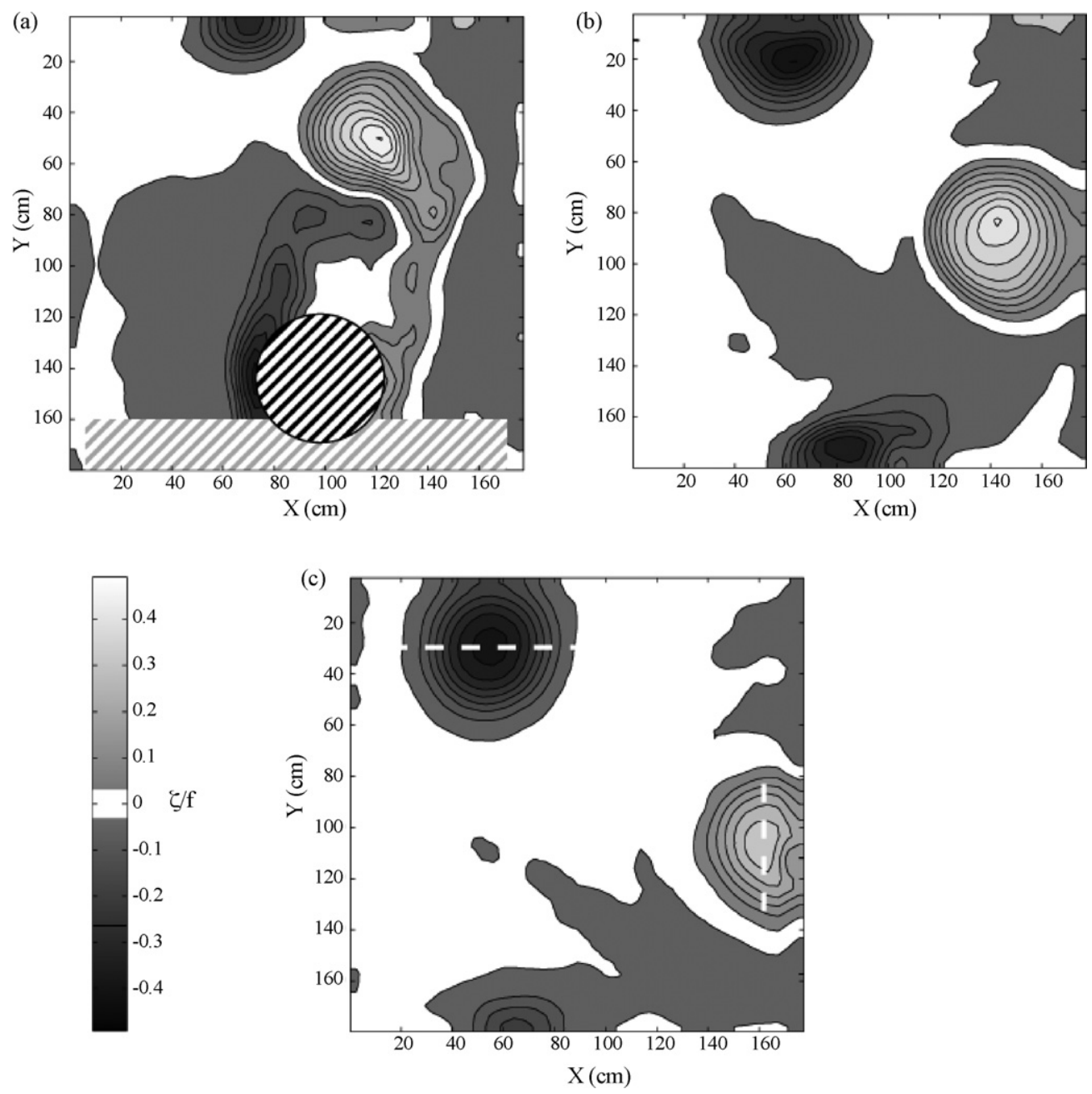

Fig. 8. Relative vorticity $\omega / f$ of the PIV1 experiment ( $R o \simeq 0.4, R e \simeq 4000$, and $B u \simeq 6.5$ ), at (a) $t=0 \mathrm{~s}$, (b) $t \simeq 400 \mathrm{~s}$ and (c) $t \simeq 1500 \mathrm{~s}$. The period of rotation of the platform is $T_{0}=160 \mathrm{~s}$ and the cylinder is drifted at constant velocity $V=0.8 \mathrm{~cm} \mathrm{~s} \mathrm{~s}^{-1}$. Each isolines are separated by $0.05 \mathrm{U}$.

detachment of the lateral boundary layer leading to free shear layers are visible in Fig. 8(a). The formation of elliptical structures just behind the obstacle in shown in Fig. 8(a). Then, these transient elliptical structures evolve quickly to axisymmetric vortices and a quasisteady vortex street is reached in the final stage Fig. 8(b) and (c). In a perfect two-dimensional flow, the region of vorticity extrema corresponds to closed streamlines where passive tracers are trapped. Typical velocity and vorticity profiles corresponding to the dashed-lines cross-sections in Fig. 8(c) are given respectively in Figs. 10(a) and 11(a) We first note that cyclonic and anticyclonic eddies are strictly identical in shape and intensity. A typical vortex diameter (distance between velocity extremas) scales with the cylinder diameter. Besides, the relative intensity $\zeta_{\max } / f \simeq 0.4$ of these vortices is of the same order of magnitude than the global Rossby number. For this small Rossby number, according to both the dye visualization and the quantitative PIV measurements, there is no evidences of cyclone-anticyclone asymmetry or any three-dimensional instability. Moreover, we fit the vorticity profile of these eddies with the standard profiles (Carton et al., 1989; Stegner and Dritschel, 2000): $\zeta(r)=\zeta_{\max }\left(1-(1 / 2)\left(r / r_{0}\right)^{p}\right) \exp \left(-\left(r / r_{0}\right)^{p}\right)$ where $p$ is the 

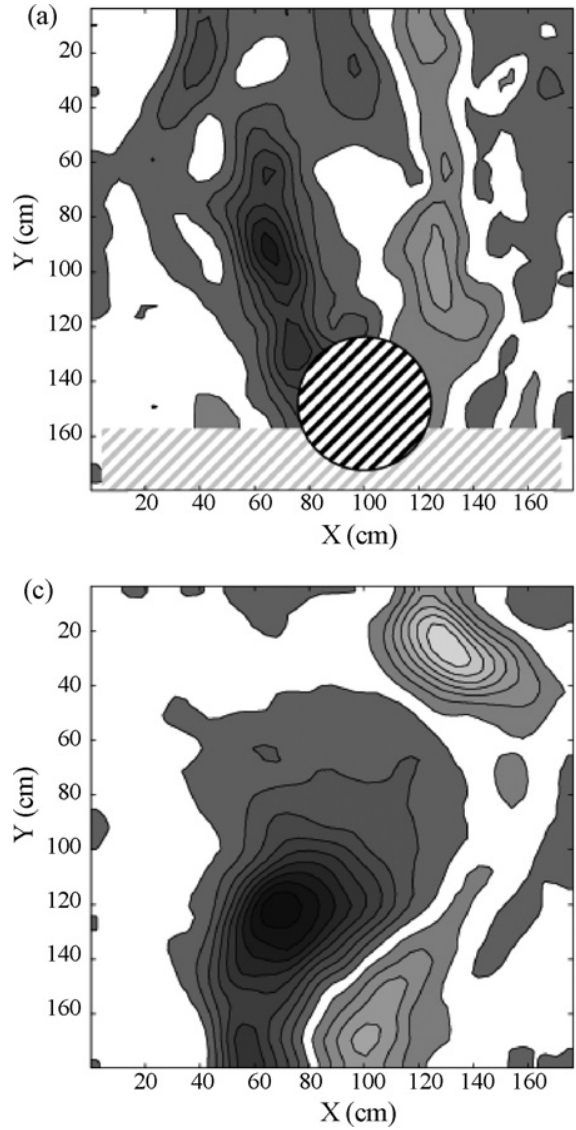

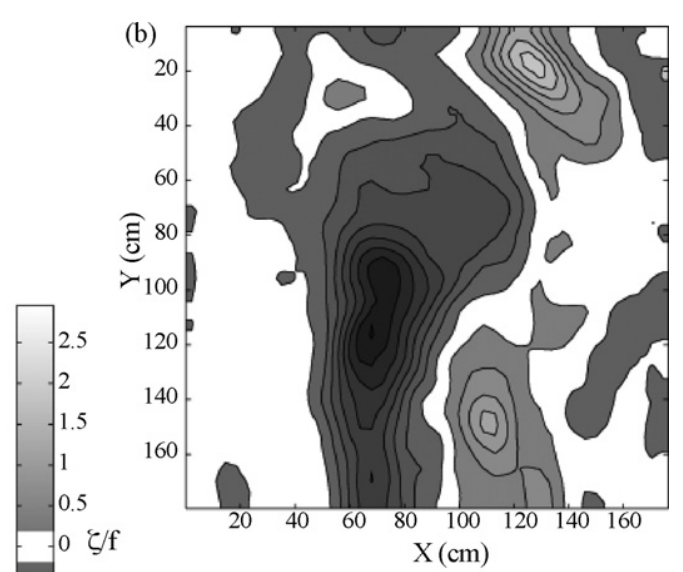

-0.5
-1
-1.5
-2
-2.5

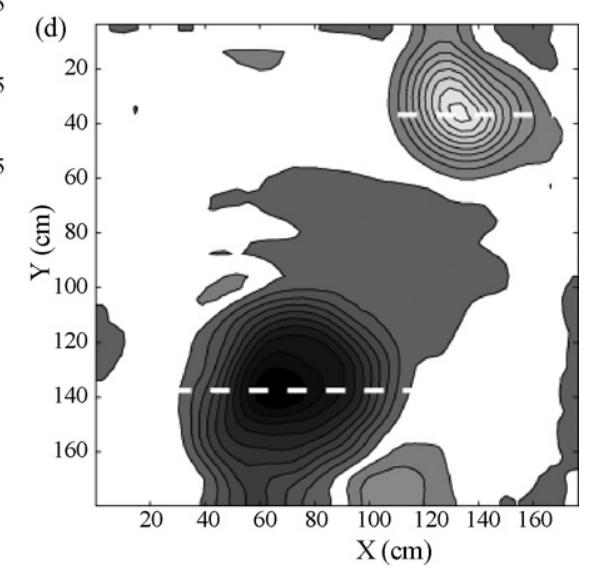

Fig. 9. Relative vorticity $\omega / f$ of PIV 2 experiment $(R o \simeq 2, R e \simeq 20,000$, and $B u \simeq 6.5$ ), at (a) $t=0 \mathrm{~s}$, (b) $t \simeq 20 \mathrm{~s}$, (c) $t \simeq 40 \mathrm{~s}$, and (d) $t \simeq 60 \mathrm{~s}$. The period of rotation of the platform is $T_{0}=160 \mathrm{~s}$ and the cylinder is drifted at constant velocity $V=4 \mathrm{~cm} \mathrm{~s}^{-1}$. Each isolines are separated by $0.3 \mathrm{U}$.

steepness parameter. According to the solid line in Fig. 11(a) the profile of both the cyclonic and anticyclonic eddies $(p \simeq 1.9)$ in the vortex street are close to Gaussian vortices $p=2$. Steep vorticity profiles $p \geq p_{c}$ (where $p_{c}$ is the critical steepness) are unstable to barotropic shear instability, the initially circular vortex will lead to a tripolar structure or even a dipolar splitting (Carnevale and Kloosterziel, 1994). The critical steepness is about $p_{c} \simeq 1.85$ for inviscid two-dimensional vortices (Carton et al., 1989) and according to Stegner and Dritschel (2000) slightly above $p \simeq 2.1$ in a shallow-water configuration where $B u=6.5$. Hence, the vortices in this small Rossby number wake are stable to both the three-dimensional inertial-centrifugal instability and the two-dimensional shear instability. The vorticity decay (Fig. 12(a)) is mainly controlled by the viscous dissipation.

In the numerical study of Dong et al. (2007) using the Regional Oceanic Model System (ROMS) a selective destabilisation of anticyclonic vorticity region occurs even for small value of the global Rossby number $R o=0.1$. Small-scale disturbances are clearly visible in the vorticity field. However, if the global Rossby number is small the amplitude of the relative vorticity $|\zeta / f|$ appears to be finite and locally larger than unity, which is not the case in the experiment PIV1. Indeed, the Reynolds number in the experiment is about $R e \simeq 4000$ while in the numerical study there is no explicit viscous dissipation for the horizontal momentum and the boundary layer thickness around the island is controlled by a land-mask algorithm. This boundary layer seems to be much larger in our laboratory experiments than in the numerical investigations of Dong et al. (2007). Hence, it could explain why, for a given Rossby 

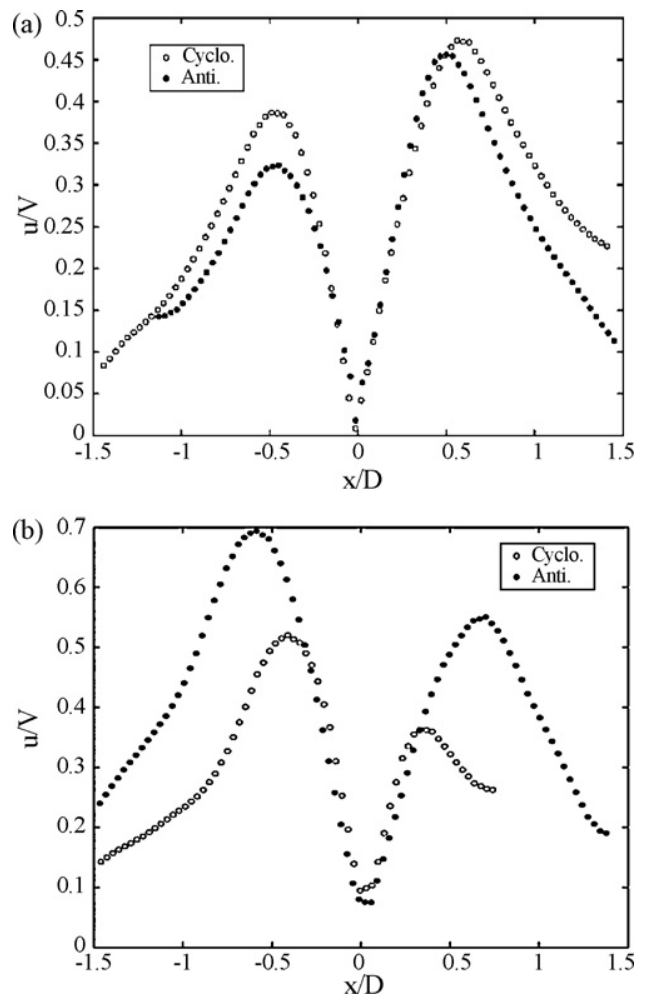

Fig. 10. Velocity profiles of cyclonic and anticyclonic vortices for (a) PIV1 experiment, and (b) PIV2 experiment, from lines represented in Figs. 8(c) and 9(d), respectively.

number, the vorticity amplitude in the island wake is weaker in the experiments than in the numerical model.

\subsubsection{Finite Rossby number wake (PIV2)}

We next measured the vorticity field for a finite Rossby number regime ( $R o=2, R e=20,000, B u=$ 6.5) where small-scale three-dimensional instability is expected to occurs. This case corresponds to an AS dye pattern (Fig. 6) described in the previous section. As for the standard two-dimensional wake, two detached boundary layers are formed just behind the cylinder (Fig. 9 (a)). Very quickly, the anticyclonic vorticity region extends on a larger area than the cyclonic vorticity region. The anticyclonic eddies shedded in the lee of the obstacle are bigger than their cyclonic counterpart (Fig. 9(d)). The velocity and vorticity profiles corresponding to the dashed-lines cross-sections in Fig. 9(d) are given respectively in Figs. 10(b) and 11(b).

The relative vorticity amplitude for both the cyclonic and the anticyclonic eddy reached finite values $|\zeta / f| \simeq 3-3.5$ larger than the global Rossby number $R o \simeq 2$. On one hand, the size and the vorticity profile of the cyclonic eddy is similar to the small Rossby case discussed above. On the other hand, for the anticyclonic eddy the relative diameter is $50 \%$ larger than the small Rossby case. Hence, the threedimensional perturbations, visualized by passive dye tracers in Figs. 4 and 6, seems to enhance the horizontal diffusion of vorticity. However, surprisingly, unlike the AS dye pattern, we did not observe in the PIV2 experiment any vortex splitting or stretching in the vorticity field. The dispersion and transport of passive tracers (dye) and active tracers (vorticity) are very different especially when dissipation caused by small-scale instability, or tilting of vorticity tube induced by three-dimensional motion, breaks the lagrangian conservation of the vertical component of vorticity or potential vorticity. According to Fig. 3 in Dong et al. (2007), strong stretching of anticyclonic vorticity could occurs 

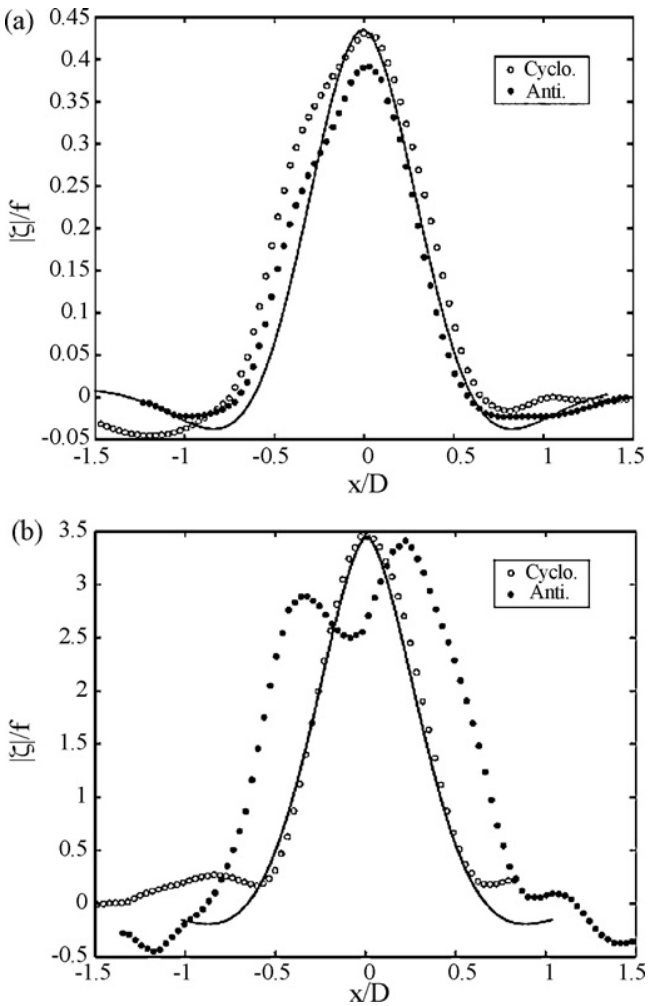

Fig. 11. Vorticity profiles of cyclonic and anticyclonic vortices for (a) PIV1 experiment, and (b) PIV2 experiment, from lines represented in Figs. 8(c) and 9(d), respectively. The vorticity profiles are fitted with standard profiles (Section 3.2.1) having a steepness parameter equal to (a) $p=1.9$ and (b) $p=1.6$.

in shallow-water wake when the relative vorticity exceed unity. However, even if large anticyclonic filaments are expelled, an axisymmetric core of anticyclonic vorticity remains at the final stage. Due to the limited PIV resolution $(130 \times 130$ grid points in comparison with $720 \times 360$ numerical grid) we could smooth out the stretched filament and detect only the main anticyclonic core.

According to Figs. 11(b) and 12(b), the vertical vorticity amplitude does not seem to be affected by the small-scale instability. Indeed, there is no difference between the peak vorticity of cyclonic or anticyclonic eddies when they are formed just behind the cylinder (Fig. 11(b)). Moreover, starting with equal initial amplitude, both anticyclonic and cyclonic vortices decay at the same rate (Fig. 12). Unlike previous studies (Stegner et al., 2005; Dong et al., 2007) there is no evidence here of a strong vorticity dispersion in the core of anticyclonic eddies, induced by the small-scale turbulent motion, leading quickly to the marginal stability limit $|\zeta / f|=1$. Nevertheless, according to Fig. 13 the anticyclonic velocity profile will be strongly smoothed unlike the cyclonic counterpart. Similar results were found in the numerical investigations of Kloosterziel et al. (2007): the core of the unstable anticyclonic vortex (i.e. the maximum vorticity) is weakly affected by the three-dimensional disturbances while the maximum velocity is strongly reduced. The dashed lines in Fig. 13(b) delimits the initial region of negative $\Phi_{R}<0$ at $t=0.5 T_{0}$. Hence, a significant kinetic energy dissipation occurs in an annular region, surrounding the vortex core, where the Rayleigh discriminant $\Phi_{R}$ is negative.

\subsubsection{General instability criteria}

In order to localize the regions where three-dimensional instabilities could occur in the shallowwater wake, we applied the general inviscid criterion proposed by Leblanc and Cambon (1997) on the 

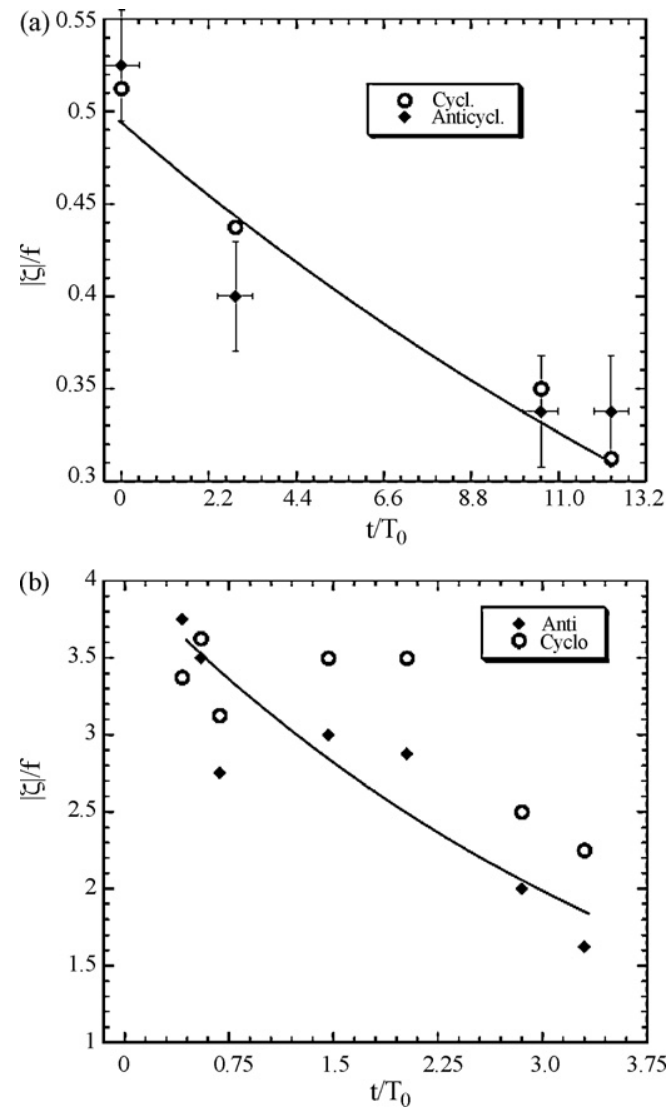

Fig. 12. Temporal evolution of the vorticity measured in the core of the anticyclonic and cyclonic vortices, for (a) PIV1 and (b) PIV2 experiments. A fit with exponential decay is indicated, with a decay rate about $25 T_{0}$ for (a) and $5 T_{0}$ for (b) ( $10 T_{0} R 0^{-1}$ for both).

velocity field of the two experiments PIV1 and PIV2. Using a local short-wave stability analysis for specific pressureless perturbations ${ }^{2}$, these authors derive a sufficient condition for instability. Rotating plane flows will be unstable to three-dimensional perturbations if, in cartesian coordinates, the scalar:

$$
\begin{aligned}
\Phi_{L C}(x, y) & =-\frac{1}{2} \underline{\underline{S}}: \underline{\underline{S}}+\frac{1}{4} W_{t} \cdot W_{t} \\
& =-\frac{1}{2}\left[\left(\partial_{x} u_{x}\right)^{2}+\left(\partial_{y} u_{y}\right)^{2}\right]+\left(2 \Omega_{0}-\partial_{y} u_{x}\right)\left(2 \Omega_{0}+\partial_{x} u_{y}\right)
\end{aligned}
$$

is negative somewhere in the flow domain. The pressureless perturbations are the leading order approximation for short-wavelength perturbations $k_{z} L \gg 1$, in other words when the typical vertical wavelength of the unstable mode is much smaller than the characteristic horizontal length-scale $L$ of the plane flow. Hence, according to the small vertical to horizontal aspect ratio of our experiments $\alpha=h / L \simeq 0.1$ which restrict the vertical wavelength of any disturbance, the simplified short-wave stability analysis should be relevant for shallow-water wakes. We assume here that the growth of small-scale three-dimensional perturbations is faster than the evolution of the large-scale flow. Hence,

\footnotetext{
${ }^{2}$ For basic flows exhibiting a characteristic length scale, these pressureless instabilities consist in short-wave eigenmodes localized along the streamlines (Leblanc and Cambon, 1997).
} 

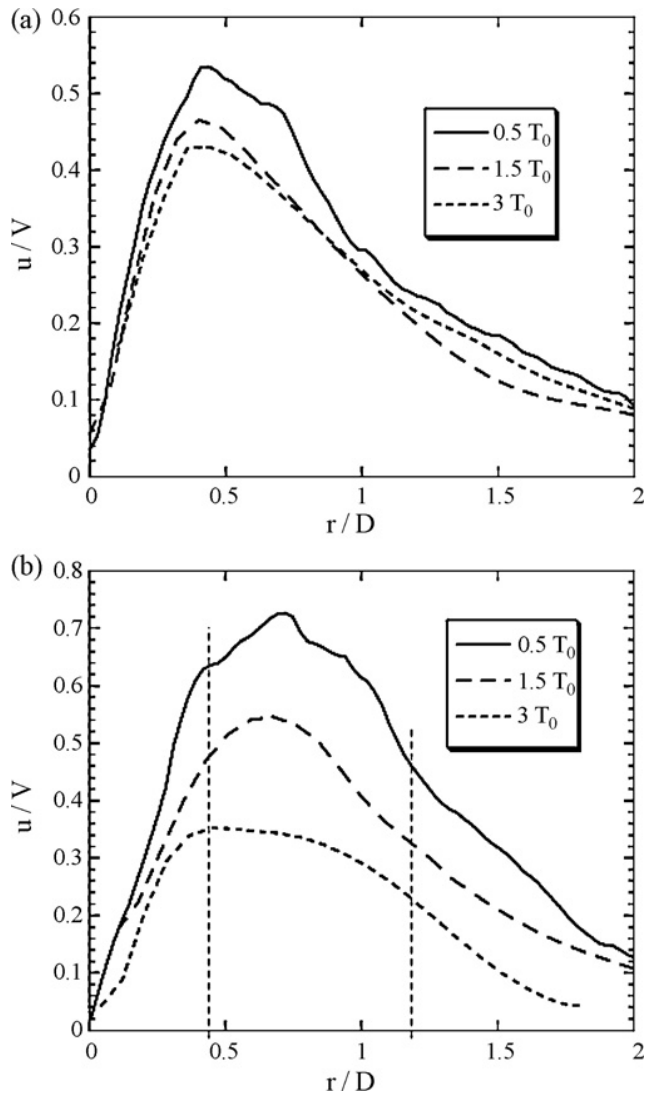

Fig. 13. Temporal evolution of the velocity profile measured in (a) cyclonic and (b) anticyclonic vortices, for PIV2 experiment. The dashed lines in (b) delimits the region where $\Phi_{R}(r)$ is negative at $t=0.5 T_{0}$.

the scalar $\Phi$ is expected to give quantitative information on the location of three-dimensional disturbances and their relative growth rates, if there is a time-scale separation between the unstable growth and the mean flow evolution. Besides, this instability criterion combines the inertial, the centrifugal and the elliptical instability, which can all be active in the cylinder wake.

For unidirectional flows the scalar $\Phi_{L C}$ recover the Rayleigh discriminant $\Phi_{i}=f(f+\zeta)$ (for parallel flows) or $\Phi_{R}(r)=\left(f+2 v_{\theta} / r\right)(f+\zeta)$ (for circular vortices). According to previous stability analysis (Billant and Gallaire, 2005; Kloosterziel et al., 2007) the growth rates $\sigma$ of unstable three-dimensional perturbations are bounded by the square root of the Rayleigh discriminant $\sigma \leq \sqrt{-\Phi_{\max }}$. Moreover, the growth of small amplitude disturbances are initially localized in the region $\Phi<0$. In the limit of the short-wavelength approximation (Leblanc and Cambon, 1997) theses properties of the Rayleigh discriminant, derived for unidirectional flows, could be extended to shallow-water flows. Hence, the scalar $\Phi_{L C}$ is expected to give quantitative information on the location of three-dimensional disturbances and their relative growth rates.

We plot in Fig. 14 the value of $\Phi_{L C}$ in the flow domain at various stages of the finite Rossby number wake experiment PIV2. The snapshots of Fig. 14 are plotted at the same times than the vorticity maps in Fig. 9 for a better comparison. Regions with negative values of $\Phi_{L C}$ are emphasized with gray levels, while positive values region remain white. The first unstable region which appears in the wake is the detached anticyclonic boundary layer cylinder Fig. 14(a). Then, this unstable tongue is stretched and tilted in the lee of the cylinder Fig. 14(b) and (c). When an anticyclonic and almost axisymmetric vortex is formed, the Leblanc and Cambon criterion indicates an unstable annular region surrounding 

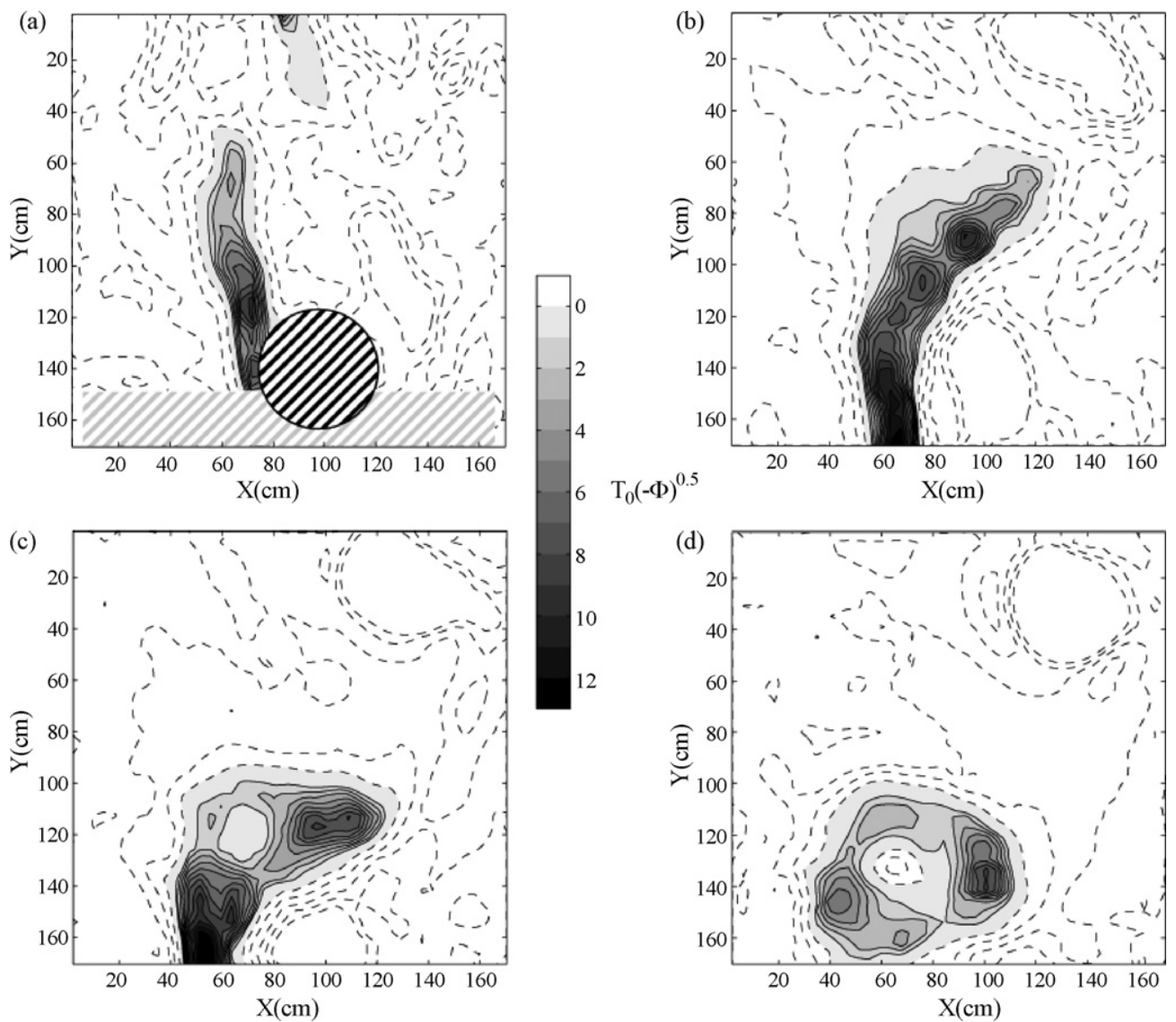

Fig. 14. Representations of the Leblanc and Cambon criterion, for the PIV2 experiment. Each isolines are separated by $1 \mathrm{U}$. Complex isolines are dashed.

the vortex core Fig. 14(d). This region is similar to the unstable region predicted by the generalized Rayleigh criterion for centrifugal instability. However, high negative values are reached in the initial stage of vortex shedding when the boundary layer detached from the cylinder and rolls up into an elliptical structures. Hence, it indicates that the three-dimensional unstable perturbations will first appear in the detached boundary layer and the stretched elliptical structure, in the near wake. Besides, the unstable regions predicted by $\Phi_{L C}$ Fig. 14(a), (b) and (c) are in good agreement with the location of small-scale disturbances revealed by the black dye tracer in Fig. 6(a) and (b).

For the small Rossby number experiment PIV1, $\Phi_{L C}$ is positive in all the flow domain. This results do not guarantee the linear stability of the flow to all three-dimensional perturbations, indeed the Leblanc and Cambon criterion is only a sufficient criterion for instability. Nevertheless, the qualitative dye visualizations do not reveal here any small-scale instability.

\subsubsection{Detached boundary layer}

In addition to the near and the far wake velocity measurements, we performed, for the experiment PIV2, zoomed visualizations of the detached boundary layer in the frame of the moving cylinder. Due to the shadow induced by the cylinder in the horizontal laser sheet, we could extract quantitative measurements on the anticyclonic side of the cylinder only. The $720 \times 576$ pixels resolution of the video camera attached to the cylinder provides a velocity grid resolution of $\delta x=1.6 \mathrm{~cm}$. We were then able to resolve the strong velocity shear of the detached boundary layer Fig. 15 (a). According to Fig. 15(b) 

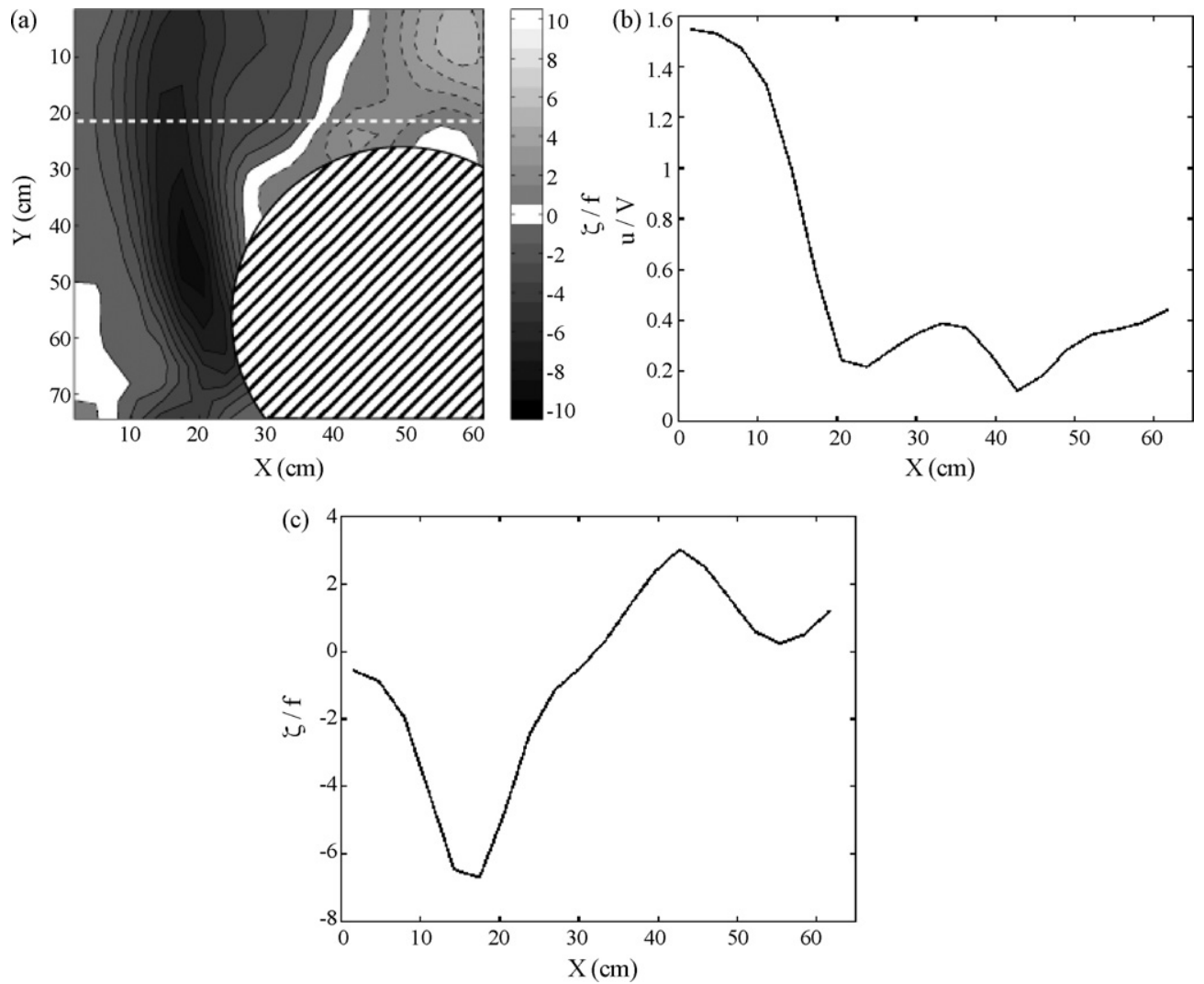

Fig. 15. Boundary Layer for the PIV2 experiment; (a) vorticy field; (b) and (c) velocity and vorticity profiles on the dashed line.

the relative vorticity in this anticyclonic parallel shear layer could exceed unity with typical values $|\zeta / f| \simeq 6-7$. These vorticity values are at least two times larger than the PIV measurements (Fig. 9(a)) obtained with a larger visualization window and a smaller velocity grid resolution $(\delta x=2.4 \mathrm{~cm})$. Hence, specific high-resolution PIV measurements are needed to capture accurately the thin and intense shear layers just behind the cylinder. Inertial instability of such parallel shear layer is expected to trigger the growth of three-dimensional perturbations and indeed, according to the dye visualisation, small-scale disturbances are already visible in the detached boundary layer. The general instability criterion of Leblanc and Cambon also capture this thin region of inertial instability (Fig. 16).

\subsubsection{A supercritical wave-vortex wake (PIV3)}

For high drifting speed the gravitational Froude $F r=V / \sqrt{g^{* h}}$ number could exceed unity. In this case, the flow around the cylinder will become locally supercritical and we could expect hydraulics jumps or high amplitude lee waves. The experiment PIV3 $(R o=3, R e=30,000, B u=6.5, F r=1.1)$ corresponds to such a supercritical case. According to the vorticity field (Fig. 17), just behind the cylinder, we observe parallel shear layers without any vortex formation. Further, down in the wake (few diameters behind the obstacle) the parallel shear flow is destabilized and a vortex street emerges. However, the relative vorticity of this vortex street $|\zeta / f| \simeq 1$ is here much smaller than the Rossby number $R o \simeq 3$. Such vorticity amplitude corresponds to the threshold of three-dimensional instabilities (inertial, elliptical or centrifugal) and therefore this supercritical wake is marginally stable to the small-scale instability described above. Hence, there is no distinction, in size or in intensity, between cyclonic and anticyclonic eddies in the vortex street (Fig. 17(d)). 


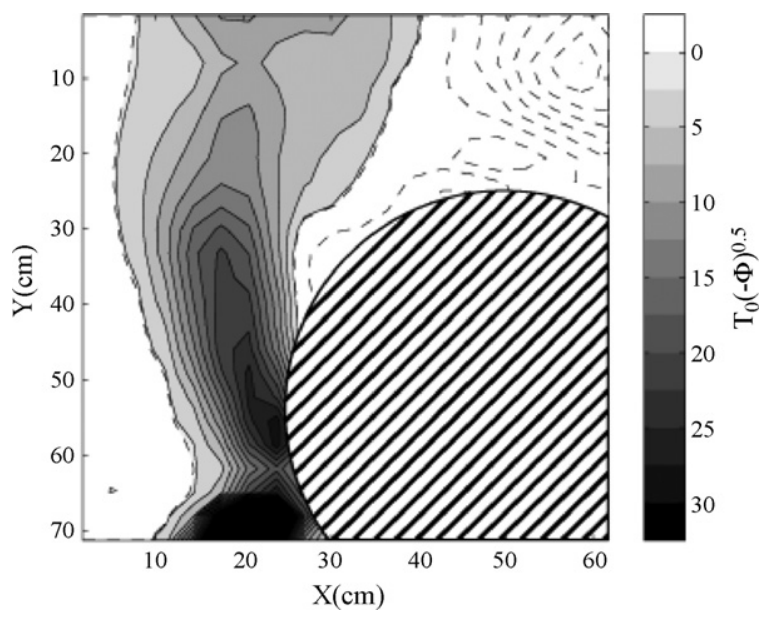

Fig. 16. Representations of the Leblanc and Cambon criterion, for the BL of PIV2 experiment. Each isolines are separated by $2.5 \mathrm{U}$. Complex isolines are dashed.
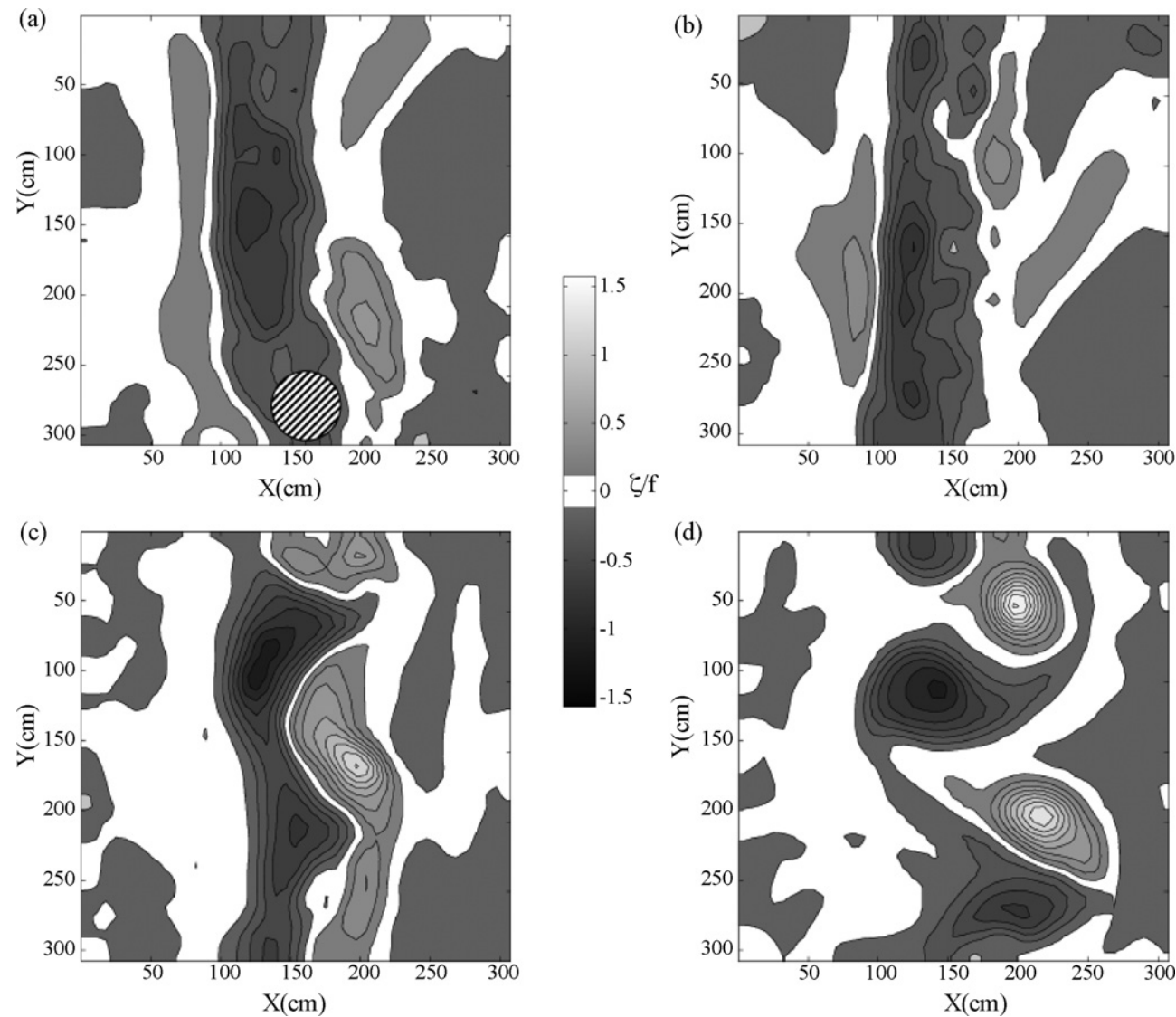

Fig. 17. Relative vorticity $\omega / f$ of PIV 3 experiment ( $R o \simeq 3, R e \simeq 30,000, B u \simeq 6.5$, and $F r \simeq 1.1$ ), at (a) $t=0 \mathrm{~s}$, (b) $t \simeq 20 \mathrm{~s}$, (c) $t \simeq$ $40 \mathrm{~s}$, and (d) $t \simeq 60 \mathrm{~s}$. The period of rotation of the platform is $T_{0}=160 \mathrm{~s}$, the cylinder is drifted at constant velocity $V=4 \mathrm{~cm} \mathrm{~s}^{-1}$. The gray-scale table is the same for the four images, and each isolines are separate by $0.16 \mathrm{U}$. 


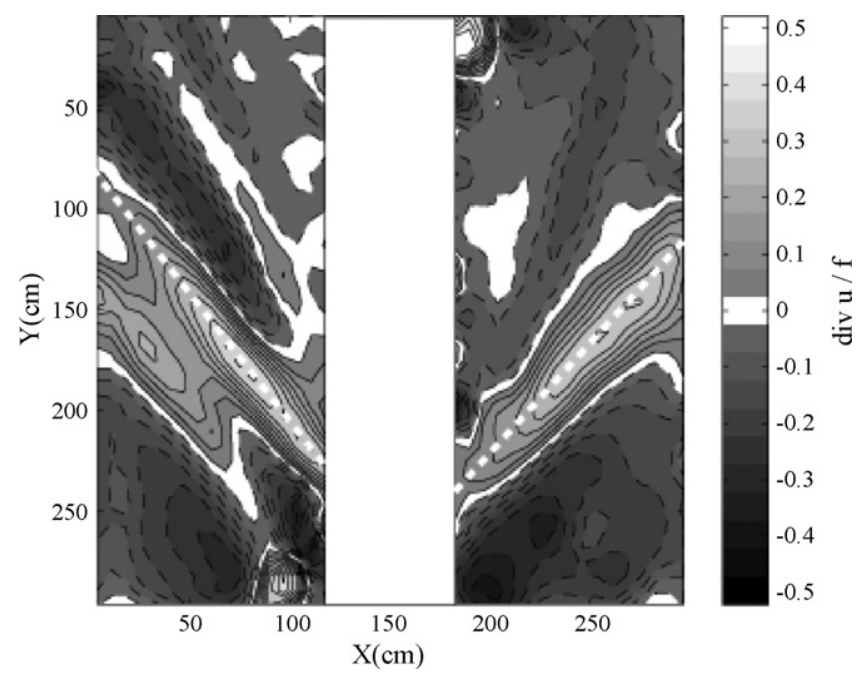

Fig. 18. Relative divergence $\operatorname{div}(u) / f$ of PIV 3 experiment $(R o \simeq 3, R e \simeq 30,000, B u \simeq 6.5$, and $F r \simeq 1.1)$, just behind the cylinder (corresponding to Fig. 17(b)). Each isolines are separate by $0.05 \mathrm{U}$, and negative isolines are dashed.

Recent investigations of Johnson et al. (2006) were devoted to nonlinear wave wakes forced by a travelling obstacle in a shallow layer of fluid. Rotating and non-rotating cases were compared each other's. They used the same experimental setup as us on the LEGI-Coriolis platform but with a rectangular dome obstacle, which partially enters into the surface layer. Such smoothed obstacle is expected to induce, if any, a weaker flow separation than our vertical cylinder. This laboratory setup was build to reproduce the interactions between an isolated mountain and an upstream wind in a shallow atmospheric boundary layer under a low-level inversion. Hence, this experiment slightly differs in its conception and its applications than ours. A supercritical case $(R o=1.5, R e=15,000, B u=4, F d=$ 1.25) is presented in Fig. 5 of Johnson et al. (2006). A symmetric V-shaped wave pattern extending laterally with a $\sim 45^{\circ}$ angle is detected in the near wake of the obstacle. This wave-wake is very close to our observations for the supercritical experiment PIV3, as it can be seen in the divergence of the horizontal velocity field (Fig. 18).

\section{Conclusion}

The purpose of this work was to investigate the influence of the vertical confinement imposed by the shallow-water configuration on the three-dimensional destabilization of vortex streets behind oceanic islands. These experiments have shown that the transient three-dimensional instabilities (inertial, elliptical or centrifugal) do persist in a shallow-water configuration, in other words when the vertical to horizontal aspect ratio is small $\alpha \ll 1$. By means of dye visualizations, we quantify in the $(R o-R e)$ parameter space, the specific dispersion regimes induced by the small-scale unstable perturbations. For finite Rossby number $(R o>0.8)$ and moderate Reynolds numbers $(R e \sim 5000-10,000)$ the passive dye tracers could be strongly mixed within the core of anticyclonic vortices while for higher Reynolds numbers $(R e>15,000)$ the passive tracers initially released in the anticyclonic island boundary layer could be strongly stretched and mixed in the near wake flow.

The vertical mixing is known to enhance biological productivity in the vicinity of island. It is well know that the cyclo-geostrophic balance induce isopycnals upwelling in the centre of cyclonic eddies. Stable cyclonic eddies are then expected to trapped cold water with increased organic carbon and larger concentration of phytoplankton. Our study suggests that anticyclonic vorticity regions could also play a significant role in the vertical mixing and the enhancement of biological activity. In agreement with recent numerical study (Dong et al., 2007) our laboratory investigations show that when the local vorticity is high enough (finite Rossby number) the anticyclonic boundary layer and the detached 
anticyclones in the near wake flow are expected to strongly mix and stir the upper ocean behind isolated islands.

The dye visualisations reveal that the typical horizontal wavelength of three-dimensional perturbations is much smaller than the horizontal scales such as the island diameter. Hence, unlike the previous studies with large $\alpha \gg 1$ or finite $\alpha \simeq 1$ aspect ratio, the vertical confinement tend to restrict here both the vertical and the horizontal scales of the unstable modes. According to qualitative dye visualisation the typical horizontal scale of the unstable disturbances seems to be fixed by the upper layer thickness (few centimetres) rather than the size of the unstable region defined by the Rayleigh discriminant (ten of centimetres). Hence, both the vertical and the horizontal resolution of regional oceanographic models should be increased to capture accurately this small-scale and three-dimensional instability. The increase of enstrophy for higher grid resolution, indicating the emergence of smaller unstable perturbations, was clearly shown by the numerical study of Dong et al. (2007). However, the precise wavelength selection (vertical and horizontal) and the hydrostatic or non-hydrostatic nature of such type of instability are still under discussion and further investigations are needed.

The growth of such small-scale perturbations is expected to be strongly controlled and restricted by the dissipative processes and the Reynolds number. Quantitative PIV measurements have shown that intense negative vorticity $(\zeta / f \simeq-2<-1)$ could hold for several rotation periods. Unlike the deep water case $(\alpha=10, R e=150$ ), the unstable flow does not reach quickly the marginal stability limit in the shallow-water configuration $(\alpha=0.1, R e=5000)$. Hence, a smaller aspect ratio of the wake flow induces a less efficient diffusion of the intense anticyclonic vorticity even if it significantly smoothed the maximum velocity. The small-scale disturbances strongly affect the annular region where the Rayleigh discriminant is negative but seems to have a much weaker influence on the vortex core. Finally, for high Froude numbers, when the flow becomes supercritical, due to the generation of high amplitude wave wake the vortex street intensity is strongly reduced.

\section{References}

Afanasyev, Y.D., 2002. Experiments on instability of columnar vortex pairs in rotating fluid. Geophys. Astro. Fluid 96 (1), 31-48.

Afanasyev, Y.D., Peltier, W.R., 1998. Three-dimensional instability of anticyclonic swirling flow in rotating fluid: laboratory experiments and related theoretical predictions. Phys. Fluids 10 (12), 3194-3202.

Aristegui, J., Sangra, P., Hernandezleon, S., Canton, M., Hernandezguerra, A., 1994. Island-induced eddies in the canary-islands. Deep-Sea Res. Pt. I 41 (10), 1509-1525.

Bartello, P., Metais, O., Lesieur, M., 1994. Coherent structures in rotating 3-dimensional turbulence. J. Fluid Mech. 273, 1-29.

Bayly, B.J., 1986. Three-dimensional instability of elliptic flow. Phys. Rev. Lett. 57 (17), 2160-2163.

Billant, P., Gallaire, F., 2005. Generalized rayleigh criterion for non-axisymmetric centrifugal instabilities. J. Fluid Mech. 542, 365-379.

Boyer, D.L., Kmetz, M.L., 1983. Vortex shedding in rotating flows. Geophys. Astro. Fluid 26, 51-83.

Caldeira, R.M.A., Groom, S., Miller, P., Pilgrim, D., Nezlin, N.P., 2002. Sea-surface signatures of the island mass effect phenomena around Madeira island, Northeast Atlantic. Remote Sens. Environ. 80, 336-360.

Caldeira, R.M.A., Marchesiello, P., 2002. Ocean response to wind sheltering in the southern California bight. Geophys. Res. Lett. $29,1-4$

Caldeira, R.M.A., Marchesiello, P., Nezlin, N.P., DiGiacomo, P.M., McWilliams, J.C., 2005. Island wakes in the southern California bight. J. Geophys. Res., 110.

Cambon, C., Benoit, J.P., Shao, L., Jacquin, L., 1994. Stability analysis and large-eddy simulation of rotating turbulence with organized eddies. J. Fluid Mech. 278, 175-200.

Carnevale, G.F., Kloosterziel, R.C., 1994. Emergence and evolution of triangular vortices. J. Fluid Mech. 259, 305-331.

Carton, X., Flierl, C.R., Polvani, L., 1989. The generation of tripoles from unstable axisymmetric isolated vortex structure. Europhys. Lett. 9, 339-344.

Chavanne, C., 2007. Observations of vortices and vortex rossby waves in the lee of an island. In: Proceeding, CFM Grenoble.

Coutis, P.F., Middleton, J.H., 1999. Flow-topography interaction in the vicinity of an isolated, deep ocean island. Deep-Sea Res. Pt. I 46 (9), 1633-1652.

Dong, C.M., McWilliams, J.C., Shchepetkin, A.F., 2007. Island wakes in deep water. J. Phys. Oceanogr. 37 (4), $962-981$.

Emanuel, K.A., 1979. Inertial stability and mesoscale convective systems. Bull. Am. Meteorol. Soc. 60 (5), 527-1527.

Hasegawa, D., Yamazaki, H., Lueck, R.G., Seuront, L., 2004. How islands stir and fertilize the upper ocean. Geophys. Res. Lett. 16, 31.

Heywood, K.J., Stevens, D.P., Bigg, G.R., 1996. Eddy formation behind the tropical island of aldabra. Deep-Sea Res. Pt. I 43 (4), $555-578$.

Johnson, E.R., Esler, J.G., Rump, O.J., Sommeria, J., Vilenski, G.G., 2006. Orographically generated nonlinear waves in rotating and non-rotating two-layer flow. P. R. Soc. A 462, 3-20.

Johnson, J.A., 1963. The stability of shearing motion in a rotating fluid. J. Fluid Mech. 17 (3), 337-352.

Kloosterziel, R.C., Carnevale, G.F., Orlandi, P., 2007. Inertial instability in rotating and stratified fluids: barotropic vortices. J. Fluid Mech. 583, 379-412. 
Kloosterziel, R.C., Carnevale, G.F., 2008. Vertical scale selection in inertial instability. J. Fluid Mech. 594, 249-269.

Kloosterziel, R.C., VanHeijst, G.J.F., 1991. An experimental study of unstable barotropic vortices in a rotating fluid. J. Fluid Mech. 223, 1-24.

Le Bars, M., Le Dizès, S., Le Gal, P., 2007. Coriolis effects on the elliptical instability in cylindrical and spherical rotating containers. J. Fluid Mech. 585, 323-342.

Le Dizès, S., 2000. Three-dimensional instability of a multipolar vortex in a rotating flow. Phys. Fluids 12 (11), 2762-2774.

Leblanc, S., Cambon, C., 1997. On the three-dimensional instabilities of plane flows subjected to coriolis force. Phys. Fluids 9 (5), 1307-1316.

Mutabazi, I., Normand, C., Wesfreid, J.E., 1992. Gap size effects on centrifugally and rotationally driven instabilities. Phys. Fluids A-Fluid 4 (6), 1199-1205.

Perret, G., Stegner, A., Dubos, T., Chomaz, J., Farge, M., 2006a. Stability of parallel wake flows in quasigeostrophic and frontal regimes. Phys. Fluids, 18.

Perret, G., Stegner, A., Farge, M., Pichon, T., 2006b. Cyclone-anticyclone asymmetry of large-scale wakes in the laboratory. Phys. Fluids, 18.

Pierrehumbert, R.T., 1986. Universal short-wave instability of two-dimensional eddies in an inviscid fluid. Phys. Rev. Lett. 57 (17), 2157-2159.

Potylitsin, P.G., Peltier, W.R., 1999. Three-dimensional destabilization of stuart vortices: the influence of rotation and ellipticity. J. Fluid Mech. 387, 205-226.

Sipp, D., Jacquin, L., 2000. Three-dimensional centrifugal-type instabilities of two-dimensional flows in rotating systems. Phys. Fluids $12,1740$.

Stegner, A., 2007. Experimental reality of geostrophic adjustment. In: Nonlinear Dynamics of Rotating Shallow Water: Methods and Advances. Elsevier.

Stegner, A., Dritschel, D.G., 2000. A numerical investigation of the stability of isolated shallow water vortices. J. Phys. Oceanogr. 30, 2562-2573.

Stegner, A., Pichon, T., Beunier, M., 2005. Elliptical-inertial instability of rotating karman vortex streets. Phys. Fluids 6, 17.

Stevens, D.E., Ciesielski, P.E., 1986. Inertial instability of horizontally sheared flow away from the equator. J. Atmos. Sci. 43 (23), 2845-2856.

Tarbouriech, L., Renouard, D., 1996. Stabilisation et destabilisation par la rotation d'un sillage turbulent. C. R. Acad. Sci. III 323 , 323-391.

Tomczak, M., 1988. Island wakes in deep and shallow water. J. Geophys. Res. 93, 5153-5154.

Wen, C.Y., Lin, C.Y., 2001. Two-dimensional vortex shedding of a circular cylinder. Phys. Fluids 13, 557.

Xie, S.P., Liu, W.T., Liu, Q.Y., Nonaka, M., 2001. Far-reaching effects of the Hawaiian islands on the pacific ocean-atmosphere system. Science 292 (5524), 2057-2060.

Yanase, S., Flores, C., Metais, O., Riley, J.J., 1993. Rotating free-shear flows. 1. Linear-stability analysis. Phys. Fluids A-Fluid 5 (11), 2725-2737. 